\title{
Fluids confined in porous media: An ideal gas in different matrices
}

\author{
W.Dong \\ Laboratoire de Chimie, UMR 5182 CNRS, Ecole Normale Supérieure de Lyon, 46, Allée \\ d'Italie, 69364 Lyon Cedex 07, France
}

Received August 16, 2007

At present, a number of models are available describing the adsorption of a fluid in a random porous medium. In this paper, some exact and analytical results are presented. All the results are obtained for an ideal gas adsorbed in various porous matrices. It is shown that the calculations are not always trivial. In some cases of complicated matrices, it is impossible to obtain any analytical results even for an ideal gas.

Key words: porous materials, confined fluids

PACS: $61.20 . G y, 61.43 . G t$

\section{Introduction}

Porous materials have important technological applications such as molecular sieve, catalyst, chemical sensor, etc. A thorough understanding of the structure of these materials as well as the behavior of substances confined in them is very helpful in designing porous materials endowed with specific properties. Much effort (both experimental and theoretical) has been devoted to the study of porous materials. In their pioneering work, Madden and Glandt proposed a very simple model for the fluid adsorption in random porous media [1]. A large number of investigations have been devoted to Madden-Glandt model [1-82]. Recently, we have proposed some sponge-like models [31,32] and a new type of templated matrix model [33]. All these models involve quenched disorders. Although this makes such systems quite complex, some exact results can be still found. In the present paper, I will give the exact results found for the adsorption of an ideal gas in a variety of models. In the next section, I deal with Madden-Glandt model. In section 3, the templated matrix model of Van Tassel will be considered. Our recent results for the hard sponge model will be presented in section 4 . In section 5 , we also present our recent results for the new templated matrix model. The concluding remarks are given in section 6 .

\section{Some general features}

We start with the discussion of some general features of the adsorption of an ideal gas in a matrix. The equations derived in this section are valid for any matrix model to be considered in this work. The fluid is denoted as species 1 and the matrix is denoted in general by species 0 . Since only an ideal gas will be considered in this work, the fluid-fluid interaction is always set to be zero, i.e.,

$$
H_{11}=0 .
$$

In what follows we show that the fluid-matrix interaction potential for all the models considered in this work can be written in the following form,

$$
H_{10}=\sum_{i=1}^{N_{1}} v_{10}\left(\mathbf{r}_{i} ; \mathbf{X}^{M}\right)
$$


where $N_{1}$ is the number of fluid particle and $\mathbf{X}^{M}$ is an abbreviated notation of the whole set of variables needed for specifying the configuration of a given matrix realization. There can be several subsets of variables completely specifying the matrix configuration, i.e.,

$$
\mathbf{X}^{M}=\left\{\mathbf{X}_{1}^{(1)}, \mathbf{X}_{2}^{(1)}, \ldots, \mathbf{X}_{M_{1}}^{(1)} ; \ldots ; \mathbf{X}_{1}^{(i)}, \mathbf{X}_{2}^{(i)}, \ldots, \mathbf{X}_{M_{i}}^{(i)} ; \ldots ; \mathbf{X}_{1}^{(m)}, \mathbf{X}_{2}^{(m)}, \ldots, \mathbf{X}_{M_{m}}^{(m)}\right\} .
$$

For example, two sets of configuration variables are needed in order to describe the templated matrices: one is needed for matrix particles and the other one for the template. In this work, all the calculations are carried out using the grand canonical ensemble. The partition function for a particular matrix realization is given by

$$
\begin{aligned}
\Xi_{1}\left(\mathbf{X}^{M}\right) & =\sum_{N_{1}=0}^{\infty} \frac{z_{1}^{N_{1}}}{N_{1} !} \int \mathrm{d} \mathbf{r}^{N_{1}} \mathrm{e}^{-\beta H_{10}}=\sum_{N_{1}=0}^{\infty} \frac{z_{1}^{N_{1}}}{N_{1} !}\left[\int \mathrm{d} \mathbf{r}_{1} \exp \left(-\beta v_{10}\left(\mathbf{r}_{1} ; \mathbf{X}^{M}\right)\right)\right]^{N_{1}} \\
& =\exp \left\{z_{1} \int \mathrm{d} \mathbf{r}_{1} \exp \left[-\beta v_{10}\left(\mathbf{r}_{1} ; \mathbf{X}^{M}\right)\right]\right\}
\end{aligned}
$$

where

$$
z_{1}=\frac{\mathrm{e}^{\beta \mu_{1}}}{\Lambda_{1}^{3}},
$$

is the fugacity of the fluid. In equation (4), $\mu_{1}$ and $\Lambda_{1}$ are respectively the chemical potential and the thermal wavelength of the fluid and $\beta=1 / k T$ ( $k$ : Boltzmann constant; $T$ : temperature). The grand potential for a particular matrix realization is given by

$$
\Omega_{1}\left(\mathbf{X}^{M}\right)=-k T \ln \Xi_{1}\left(\mathbf{X}^{M}\right)=-k T z_{1} \int \mathrm{d} \mathbf{r}_{1} \exp \left[-\beta v_{10}\left(\mathbf{r}_{1} ; \mathbf{X}^{M}\right)\right] .
$$

Taking the average over matrix realizations, one obtains,

$$
\begin{aligned}
\Omega & =\left\langle\Omega_{1}\left(\mathbf{X}^{M}\right)\right\rangle_{M}=-k T z_{1} \int \mathrm{d} \mathbf{r}_{1}\left\langle\exp \left[-\beta v_{10}\left(\mathbf{r}_{1} ; \mathbf{X}^{M}\right)\right]\right\rangle_{M} \\
& =-k T z_{1} V\left\langle\exp \left[-\beta v_{10}\left(\mathbf{r}_{1} ; \mathbf{X}^{M}\right)\right]\right\rangle_{M},
\end{aligned}
$$

where $\langle\cdots\rangle_{M}$ denotes the average over matrix realizations. When the matrix samples are statistically homogeneous, $\left\langle\exp \left[-\beta v_{10}\left(\mathbf{r}_{1} ; \mathbf{X}^{M}\right)\right]\right\rangle_{M}$ is independent of $\mathbf{r}_{1}$. That is why the integration over $\mathbf{r}_{1}$ can be carried out in equation (6). The thermodynamic pressure is defined as

$$
P=\frac{-\Omega}{V}=k T z_{1}\left\langle\exp \left[-\beta v_{10}\left(\mathbf{r}_{1} ; \mathbf{X}^{M}\right)\right]\right\rangle_{M} .
$$

The one-body fluid distribution function for a particular matrix realization is given by

$$
\begin{aligned}
\rho_{1}^{(1)}\left(\mathbf{r} ; \mathbf{X}^{M}\right) & =\left\langle\sum_{i=1}^{N_{1}} \delta\left(\mathbf{r}_{i}-\mathbf{r}\right)\right\rangle_{1}=\frac{1}{\Xi_{1}\left(\mathbf{q}^{N_{0}}\right)} \sum_{N_{1}=0}^{\infty} \frac{z_{1}^{N_{1}}}{N_{1} !} \int \mathrm{d} \mathbf{r}^{N_{1}} \mathrm{e}^{-\beta H_{10}} \sum_{i=1}^{N_{1}} \delta\left(\mathbf{r}_{i}-\mathbf{r}\right) \\
& =\frac{\exp \left[-\beta v_{10}\left(\mathbf{r} ; \mathbf{X}^{M}\right)\right]}{\Xi_{1}\left(\mathbf{q}^{N_{0}}\right)} \sum_{N_{1}=0}^{\infty} \frac{z_{1}^{N_{1}}}{\left(N_{1}-1\right) !}\left(\int \mathrm{d} \mathbf{r}_{2} \exp \left[-\beta v_{10}\left(\mathbf{r}_{2} ; \mathbf{X}^{M}\right)\right]\right)^{N_{1}-1} \\
& =z_{1} \exp \left[-\beta v_{10}\left(\mathbf{r} ; \mathbf{X}^{M}\right)\right] .
\end{aligned}
$$

Averaging over matrix realizations, we obtain the following expression for the fluid density

$$
\rho_{1}=\left\langle\rho_{1}^{(1)}\left(\mathbf{r} ; \mathbf{X}^{M}\right)\right\rangle_{M}=z_{1}\left\langle\exp \left[-\beta v_{10}\left(\mathbf{r} ; \mathbf{X}^{M}\right)\right]\right\rangle_{M}
$$


Taking the derivative of the pressure given in equation (7) with respect to the fluid chemical potential, one verifies straightforwardly that Gibbs-Duhem equation holds, i.e.,

$$
\left(\frac{\partial P}{\partial \mu_{1}}\right)_{T}=\rho_{1} .
$$

Combining equations (7) and (9), one obtains

$$
P=k T \rho_{1}
$$

Although this equation of state has formally the same form as that for a bulk ideal gas, it will be shown that $\rho_{1}$ is the averaged fluid density inside a porous matrix which depends on the matrix characteristics.

For a particular matrix realization, the exact expression of the free energy of an inhomogeneous ideal gas is well known as a functional of the fluid one-body distribution function,

$$
\Im^{\mathrm{id}}\left(\mathbf{X}^{M}\right)=\int \mathrm{d} \mathbf{r} \rho_{1}^{(1)}\left(\mathbf{r} ; \mathbf{X}^{M}\right) v_{10}\left(\mathbf{r} ; \mathbf{X}^{M}\right)+k T \int \mathrm{d} \mathbf{r} \rho_{1}^{(1)}\left(\mathbf{r} ; \mathbf{X}^{M}\right)\left\{\ln \left[\Lambda_{1}^{3} \rho_{1}^{(1)}\left(\mathbf{r} ; \mathbf{X}^{M}\right)\right]-1\right\} .
$$

Using equations (4) and (8), one can rewrite the above expression as,

$$
\Im^{\text {id }}\left(\mathbf{X}^{M}\right)=k T\left(\beta \mu_{1}-1\right) \int \mathrm{d} \mathbf{r} \rho_{1}^{(1)}\left(\mathbf{r} ; \mathbf{X}^{M}\right) .
$$

The free energy averaged over the matrix realizations is given by

$$
F^{\mathrm{id}}=\left\langle\Im^{\mathrm{id}}\left(\mathbf{X}^{M}\right)\right\rangle_{M}=k T\left(\beta \mu_{1}-1\right)\left\langle\rho_{1}^{(1)}\left(\mathbf{r} ; \mathbf{X}^{M}\right)\right\rangle_{M} V .
$$

Equations (11) and (14) clearly show that the determination of the thermodynamic properties relies essentially on the calculation of the averaged fluid density in the matrix, i.e., $\left\langle\rho_{1}^{(1)}\left(\mathbf{r} ; \mathbf{X}^{M}\right)\right\rangle_{M}$.

Different distribution functions give us the structural information. The fluid-fluid two-body distribution function for a particular matrix realization is given by clearly

$$
\begin{aligned}
& \rho_{11}^{(2)}\left(\mathbf{r}, \mathbf{r}^{\prime} ; \mathbf{X}^{M}\right)=\left\langle\sum_{i=1}^{N_{1}} \sum_{j=1, j \neq i}^{N_{1}} \delta\left(\mathbf{r}_{i}-\mathbf{r}\right) \delta\left(\mathbf{r}_{j}-\mathbf{r}^{\prime}\right)\right\rangle_{1} \\
& \quad=z_{1}^{2} \exp \left\{-\beta\left[v_{10}\left(\mathbf{r} ; \mathbf{X}^{M}\right)+v_{10}\left(\mathbf{r}^{\prime} ; \mathbf{X}^{M}\right)\right]\right\}=\rho_{1}^{(1)}\left(\mathbf{r} ; \mathbf{X}^{M}\right) \rho_{1}^{(1)}\left(\mathbf{r}^{\prime} ; \mathbf{X}^{M}\right) .
\end{aligned}
$$

The averaging over matrix realizations leads to

$$
\rho_{11}^{(2)}\left(\left|\mathbf{r}-\mathbf{r}^{\prime}\right|\right)=\left\langle\rho_{11}^{(2)}\left(\mathbf{r}, \mathbf{r}^{\prime} ; \mathbf{X}^{M}\right)\right\rangle_{M}=\left\langle\rho_{1}^{(1)}\left(\mathbf{r} ; \mathbf{X}^{M}\right) \rho_{1}^{(1)}\left(\mathbf{r}^{\prime} ; \mathbf{X}^{M}\right)\right\rangle_{M} .
$$

It is worth noting that no fluid-fluid correlation appears at the level of a particular matrix realization [see equation (15)]. Nevertheless, the fluid-fluid correlation appears after averaging over matrix realizations, i.e.,

$$
\rho_{11}^{(2)}\left(\left|\mathbf{r}-\mathbf{r}^{\prime}\right|\right) \neq \rho_{1}^{2}
$$

The correlation results from the fluid-matrix interaction and the loss of detailed structural information on the matrix related to the averaging over matrix realizations.

Now, we consider the correlation function of fluid with one set of matrix configuration variables for a particular matrix realization,

$$
\begin{aligned}
\rho_{1 m_{k}}^{(2)}\left(\mathbf{r}, \mathbf{X} ; \mathbf{X}^{M}\right) & =\left\langle\sum_{i=1}^{N_{1}} \sum_{j=1}^{M_{k}} \delta\left(\mathbf{r}_{i}-\mathbf{r}\right) \delta\left(\mathbf{X}_{j}^{(k)}-\mathbf{X}\right)\right\rangle_{1}=\left\langle\sum_{i=1}^{N_{1}} \delta\left(\mathbf{r}_{i}-\mathbf{r}\right)\right\rangle_{1} \sum_{j=1}^{M_{k}} \delta\left(\mathbf{X}_{j}^{(k)}-\mathbf{X}\right) \\
& =\rho_{1}^{(1)}\left(\mathbf{r} ; \mathbf{X}^{M}\right) \sum_{j=1}^{M_{k}} \delta\left(\mathbf{X}_{j}^{(k)}-\mathbf{X}\right) .
\end{aligned}
$$


Taking the average over matrix realizations, we obtain

$$
\rho_{1 m_{k}}^{(2)}(|\mathbf{r}-\mathbf{X}|)=\left\langle\rho_{1 m_{k}}^{(2)}\left(\mathbf{r}, \mathbf{X} ; \mathbf{X}^{M}\right)\right\rangle_{M}=\left\langle\rho_{1}^{(1)}\left(\mathbf{r} ; \mathbf{X}^{M}\right) \sum_{j=1}^{M_{k}} \delta\left(\mathbf{X}_{j}^{(k)}-\mathbf{X}\right)\right\rangle_{M}
$$

\section{Madden-Glandt model}

\section{A. The overlapping hard-sphere matrix}

The overlapping hard-sphere matrix model is sketched in figure 1. The interaction potentials between different species ( 0 and 1 denoting respectively matrix and fluid species) are as follows:

$$
\begin{gathered}
H_{00}=0, \\
H_{11}=0, \\
H_{10}=\sum_{i=1}^{N_{1}} \sum_{j=1}^{N_{0}} u_{10}\left(\left|\mathbf{r}_{i}-\mathbf{q}_{j}\right|\right),
\end{gathered}
$$

with

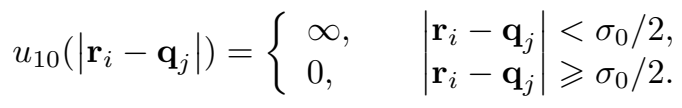

$\sigma_{0}$ in equation (23) is the diameter of the matrix hard sphere and $\mathbf{q}_{j}$ denoting the position of the $j$-th matrix particle. One obtains the partition function for a particular matrix realization by replacing $v_{10}\left(\mathbf{r}_{1} ; \mathbf{X}^{M}\right)$ in equation (3) by $\sum_{j=1}^{N_{0}} u_{10}\left(\left|\mathbf{r}_{1}-\mathbf{q}_{j}\right|\right)$,

$$
\Xi_{1}\left(\mathbf{q}^{N_{0}}\right)=\exp \left\{z_{1} \int \mathrm{d} \mathbf{r}_{1} \exp \left[-\beta \sum_{j=1}^{N_{0}} u_{10}\left(\left|\mathbf{r}_{1}-\mathbf{q}_{j}\right|\right)\right]\right\}
$$

The grand potential after taking the average over matrix realizations is as follows:

$$
\begin{aligned}
\Omega & =-k T\left\langle\ln \Xi_{1}\left(\mathbf{q}^{N_{0}}\right)\right\rangle_{0}=\frac{-k T z_{1}}{\Xi_{0}} \sum_{N_{0}=0}^{\infty} \frac{z_{0}^{N_{0}}}{N_{0} !} \int \mathrm{d} \mathbf{q}^{N_{0}} \int \mathrm{d} \mathbf{r}_{1} \exp \left[-\beta \sum_{j=1}^{N_{0}} u_{10}\left(\left|\mathbf{r}_{1}-\mathbf{q}_{j}\right|\right)\right] \\
& =\frac{-k T z_{1}}{\Xi_{0}} \sum_{N_{0}=0}^{\infty} \frac{z_{0}^{N_{0}}}{N_{0} !} \int \mathrm{d} \mathbf{r}_{1}\left(V-\frac{\pi \sigma_{0}^{3}}{6}\right)^{N_{0}}=\frac{-k T z_{1} V}{\Xi_{0}} \exp \left[z_{0}\left(V-\frac{\pi \sigma_{0}^{3}}{6}\right)\right]
\end{aligned}
$$

where

$$
z_{0}=\frac{\mathrm{e}^{\beta \mu_{0}}}{\Lambda_{0}^{3}}
$$

is the fugacity of the matrix species and $\Xi_{0}$ the partition function of the matrix which is given by,

$$
\Xi_{0}=\sum_{N_{0}=0}^{\infty} \frac{\left(z_{0} V\right)^{N_{0}}}{N_{0} !}=\mathrm{e}^{z_{0} V}
$$

Substituting equation (27) into equation (25), we obtain

$$
\Omega=-k T z_{1} V \exp \left(-\frac{\pi \sigma_{0}^{3} z_{0}}{6}\right) .
$$




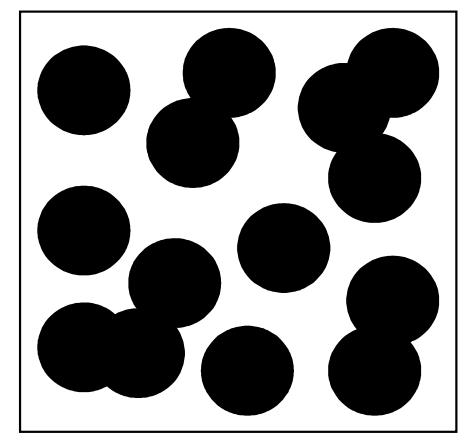

Figure 1. Sketch of the overlapping hard-sphere matrix model.

The thermodynamic pressure is given by

$$
P=\frac{-\Omega}{V}=k T z_{1} \exp \left(-\frac{\pi \sigma_{0}^{3} z_{0}}{6}\right) .
$$

Now, we consider the one-body distribution functions. For a particular matrix realization, we have

$$
\rho_{1}^{(1)}\left(\mathbf{r} ; \mathbf{q}^{N_{0}}\right)=z_{1} \exp \left[-\beta \sum_{j=1}^{N_{0}} u_{10}\left(\left|\mathbf{r}-\mathbf{q}_{j}\right|\right)\right] .
$$

The fluid density after the averaging over matrix realizations is given by

$$
\begin{aligned}
\rho_{1} & =\left\langle\rho_{1}^{(1)}\left(\mathbf{r} ; \mathbf{q}^{N_{0}}\right)\right\rangle_{0}=\frac{z_{1}}{\Xi_{0}} \sum_{N_{0}=0}^{\infty} \frac{z_{0}^{N_{0}}}{N_{0} !} \int \mathrm{d} \mathbf{q}^{N_{0}} \exp \left[-\beta \sum_{j=1}^{N_{0}} u_{10}\left(\left|\mathbf{r}-\mathbf{q}_{j}\right|\right)\right] \\
& =\frac{z_{1}}{\Xi_{0}} \sum_{N_{0}=0}^{\infty} \frac{z_{0}^{N_{0}}}{N_{0} !}\left(V-\frac{\pi \sigma_{0}^{3}}{6}\right)^{N_{0}}=z_{1} \exp \left(-\frac{\pi \sigma_{0}^{3} z_{0}}{6}\right) .
\end{aligned}
$$

Now, we will show that this result for $\rho_{1}$ has a very appealing meaning. In equation $(31), z_{1}$ is the density of a bulk ideal gas at the same chemical potential as the confined ideal gas (i.e., the gas in a reservoir outside the porous medium being in equilibrium with the confined ideal gas). It is easy to prove that $\exp \left(-\pi \sigma_{0}^{3} z_{0} / 6\right)$ is nothing else but the probability that a fluid particle is in the void space. This probability can be found by considering a given ideal gas particle (a point one) as follows. If no center of any matrix particle falls in the sphere of a diameter equal to $\sigma_{0}$ centered at the position of the given fluid particle, the considered fluid particle is obviously in the void space. This sphere will be called exclusion sphere. The probability of finding $\mathrm{M}$ matrix particles inside the exclusion sphere, $P_{e s}(M)$, is a Poisson distribution in the case of overlapping HS matrix and

$$
P_{e s}(M=0)=\exp \left(-\frac{\pi \sigma_{0}^{3} \rho_{0}}{6}\right),
$$

where $\rho_{0}$ is the density of matrix particles. Noting that $z_{0}=\rho_{0}$ for the overlapping HS matrix, we see that the density of the confined ideal gas is given by the density of the bulk ideal gas (in equilibrium with the confined one) multiplied by the probability of a fluid particle being in the void space.

Substituting equation (31) into equation (14), we obtain the following result for the Helmholtz free energy,

$$
\frac{F}{V}=k T\left(\beta \mu_{1}-1\right) z_{1} \exp \left(-\frac{\pi \sigma_{0}^{3} z_{0}}{6}\right) .
$$


In terms of densities, the free energy takes the following form,

$$
\frac{F}{V}=k T \rho_{1}\left[\ln \left(\rho_{1} \Lambda_{1}^{3}\right)+\frac{\pi \sigma_{0}^{3} \rho_{0}}{6}-1\right] .
$$

Now, we will discuss different joint two-body distribution functions. The fluid two-body distribution function is given by

$$
\begin{aligned}
\rho_{11}\left(\left|\mathbf{r}-\mathbf{r}^{\prime}\right|\right) & =\left\langle\rho_{1}^{(1)}\left(\mathbf{r} ; \mathbf{q}^{N_{0}}\right) \rho_{1}^{(1)}\left(\mathbf{r}^{\prime} ; \mathbf{q}^{N_{0}}\right)\right\rangle_{0} \\
& =\frac{z_{1}^{2}}{\Xi_{0}} \sum_{N_{0}=0}^{\infty} \frac{z_{0}^{N_{0}}}{N_{0} !} \int \mathrm{d} \mathbf{q}^{N_{0}} \exp \left\{-\beta \sum_{j=1}^{N_{0}}\left[u_{10}\left(\left|\mathbf{r}-\mathbf{q}_{j}\right|\right)+u_{10}\left(\left|\mathbf{r}^{\prime}-\mathbf{q}_{j}\right|\right)\right]\right\} \\
& =\frac{z_{1}^{2}}{\Xi_{0}} \sum_{N_{0}=0}^{\infty} \frac{z_{0}^{N_{0}}}{N_{0} !}\left[V-V_{d b}\left(\left|\mathbf{r}-\mathbf{r}^{\prime}\right|\right)\right]^{N_{0}} z_{1}^{2} \exp \left[-z_{0} V_{d b}\left(\left|\mathbf{r}-\mathbf{r}^{\prime}\right|\right)\right],
\end{aligned}
$$

where $V_{d b}\left(\left|\mathbf{r}-\mathbf{r}^{\prime}\right|\right)$ is the volume of a dumbbell with its two spheres centered at $\mathbf{r}$ and $\mathbf{r}^{\prime}$ respectively and

$$
V_{d b}(x)= \begin{cases}\frac{\pi \sigma_{0}^{3}}{3}-\frac{\pi\left(x^{3}-3 \sigma_{0}^{2} x+2 \sigma_{0}^{3}\right)}{12}, & 0 \leqslant x \leqslant \sigma_{0} \\ \frac{\pi \sigma_{0}^{3}}{3}, & x>\sigma_{0} .\end{cases}
$$

As for the one-body distribution function, the result given in equation (35) for $\rho_{11}\left(\left|\mathbf{r}-\mathbf{r}^{\prime}\right|\right)$ has also a clear probabilistic meaning. $z_{1}^{2}$ is the two-body distribution function of the bulk ideal gas in equilibrium with the confined one and $\exp \left[-z_{0} V_{d b}\left(\left|\mathbf{r}-\mathbf{r}^{\prime}\right|\right)\right]=\exp \left[-\rho_{0} V_{d b}\left(\left|\mathbf{r}-\mathbf{r}^{\prime}\right|\right)\right]$ is nothing else but the probability that the two considered particles are both in the void space. In this case, the exclusion region is a dumbbell instead of a cavity in the case of one-body distribution. The total correlation function can be obtained straightforwardly,

$$
h_{11}\left(\left|\mathbf{r}-\mathbf{r}^{\prime}\right|\right)=\frac{\rho_{11}^{(2)}\left(\left|\mathbf{r}-\mathbf{r}^{\prime}\right|\right)}{\rho_{1}^{2}}-1=\exp \left\{z_{0}\left[\frac{\pi \sigma_{0}^{3}}{3}-V_{d b}\left(\left|\mathbf{r}-\mathbf{r}^{\prime}\right|\right)\right]\right\}-1 .
$$

The matrix-fluid two-body distribution function after averaging over matrix realizations is given by

$$
\begin{aligned}
\rho_{01}(|\mathbf{q}-\mathbf{r}|) & =\left\langle\rho_{1}^{(1)}\left(\mathbf{r} ; \mathbf{q}^{N_{0}}\right) \sum_{i=1}^{N_{0}} \delta\left(\mathbf{q}_{i}-\mathbf{q}\right)\right\rangle_{0} \\
& =\frac{z_{1}}{\Xi_{0}} \sum_{N_{0}=0}^{\infty} \frac{z_{0}^{N_{0}}}{N_{0} !} \int \mathrm{d} \mathbf{q}^{N_{0}} \exp \left[-\beta \sum_{j=1}^{N_{0}} u_{10}\left(\left|\mathbf{r}-\mathbf{q}_{j}\right|\right)\right] \sum_{i=1}^{N_{0}} \delta\left(\mathbf{q}_{i}-\mathbf{q}\right) \\
& =\frac{z_{1} \mathrm{e}^{-\beta u_{10}(|\mathbf{r}-\mathbf{q}|)}}{\Xi_{0}} \sum_{N_{0}=0}^{\infty} \frac{z_{0}^{N_{0}}}{\left(N_{0}-1\right) !}\left(V-\frac{\pi \sigma_{0}^{3}}{6}\right)^{N_{0}-1} \\
& =\rho_{0} \rho_{1} \exp \left[-\beta u_{10}(|\mathbf{r}-\mathbf{q}|)\right]
\end{aligned}
$$

where equations $(27),(31)$ and $z_{0}=\rho_{0}$ were used in obtaining the last equality in equation (38).

The total matrix-fluid correlation is given by

$$
h_{01}(|\mathbf{q}-\mathbf{r}|)=\frac{\rho_{01}(|\mathbf{q}-\mathbf{r}|)}{\rho_{0} \rho_{1}}-1= \begin{cases}-1, & 0 \leqslant|\mathbf{q}-\mathbf{r}| \leqslant \sigma_{0} / 2 \\ 0, & |\mathbf{q}-\mathbf{r}|>\sigma_{0} / 2 .\end{cases}
$$

It is clear (see equation (38)) that the particles of the ideal gas are excluded from the hard core of the matrix particle and distributed uniformly outside it. The matrix-fluid and fluid-fluid correlation functions can be also obtained by using the diagrammatic method. Since there is only 
fluid-matrix interaction in the overlapping HS matrix, there can be only bonds between fluid and matrix points. For the fluid-matrix correlation, only one diagram subsists,

$$
h_{01}(|\mathbf{q}-\mathbf{r}|)=c_{01}(|\mathbf{q}-\mathbf{r}|)=\square-\bigcirc,
$$

where the bond represents the Mayer function, i.e.,

$$
f(|\mathbf{r}-\mathbf{q}|)=\exp \left[-\beta u_{10}(|\mathbf{r}-\mathbf{q}|)\right]-1 .
$$

Equation (40) together with equation (41) give the same result as equation (39). The total fluid-fluid correlation function is given by

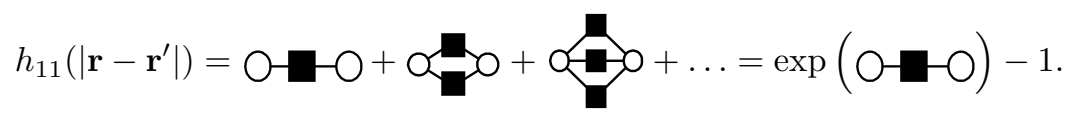

It can be easily checked that equation (42) gives the same result as equation (37). The direct fluid-fluid correlation function is given by

$$
\begin{aligned}
c_{11}\left(\left|\mathbf{r}-\mathbf{r}^{\prime}\right|\right) & =\propto-0+\infty=h_{11}\left(\left|\mathbf{r}-\mathbf{r}^{\prime}\right|\right)-0-0 \\
& =\exp \left\{z_{0}\left[\frac{\pi \sigma_{0}^{3}}{6}-V_{d b}\left(\left|\mathbf{r}-\mathbf{r}^{\prime}\right|\right)\right]\right\}-1-z_{0}\left[\frac{\pi \sigma_{0}^{3}}{6}-V_{d b}\left(\left|\mathbf{r}-\mathbf{r}^{\prime}\right|\right)\right] .
\end{aligned}
$$

Equations (42) and (43) clearly show that only the blocked part gives no vanishing contribution, i.e.,

$$
h_{11}\left(\left|\mathbf{r}-\mathbf{r}^{\prime}\right|\right)=h_{11}^{b}\left(\left|\mathbf{r}-\mathbf{r}^{\prime}\right|\right),
$$

and

$$
c_{11}\left(\left|\mathbf{r}-\mathbf{r}^{\prime}\right|\right)=c_{11}^{b}\left(\left|\mathbf{r}-\mathbf{r}^{\prime}\right|\right) .
$$

Therefore, any approximate theory in which it is imposed that $h_{11}^{b}\left(\left|\mathbf{r}-\mathbf{r}^{\prime}\right|\right)=c_{11}^{b}\left(\left|\mathbf{r}-\mathbf{r}^{\prime}\right|\right)=0$ does not make it possible to obtain the correct result for an ideal gas. The capability of recovering the ideal gas result can serve as a criterion for judging the quality of an approximate theory. It is to be pointed out that the well-know Percus-Yevick approximation (extended for confined fluids) does not satisfy this criterion.

\section{B. Hard-sphere matrix}

The HS matrix model is sketched in figure 2. The only difference from the previous model is that the matrix particles are not allowed to overlap. The interaction potentials between different species are given by

$$
\begin{gathered}
H_{11}=0, \\
H_{00}=\frac{1}{2} \sum_{i=1}^{N_{0}} \sum_{j=1, j \neq i}^{N_{0}} u_{00}\left(\left|\mathbf{q}_{i}-\mathbf{q}_{j}\right|\right),
\end{gathered}
$$

with

$$
u_{00}\left(\left|\mathbf{q}_{i}-\mathbf{q}_{j}\right|\right)=\left\{\begin{array}{l}
\infty, \\
0,
\end{array} \quad \mid \begin{array}{l}
\mathbf{q}_{i}-\mathbf{q}_{j} \\
\mathbf{q}_{i}-\mathbf{q}_{j} \mid \geqslant \sigma_{0}
\end{array}\right.
$$

The fluid-matrix interaction potential is still described by equations (22) and (23)

The partition function for a particular matrix realization is given by

$$
\Xi_{1}\left(\mathbf{q}^{N_{0}}\right)=\exp \left[z_{1} \int \mathrm{d} \mathbf{r}_{1} \exp \left(-\beta \sum_{j=1}^{N_{0}} u_{10}\left(\left|\mathbf{r}_{1}-\mathbf{q}_{j}\right|\right)\right)\right]=\exp \left[z_{1}\left(V-\frac{N_{0} \pi \sigma_{0}^{3}}{6}\right)\right] .
$$




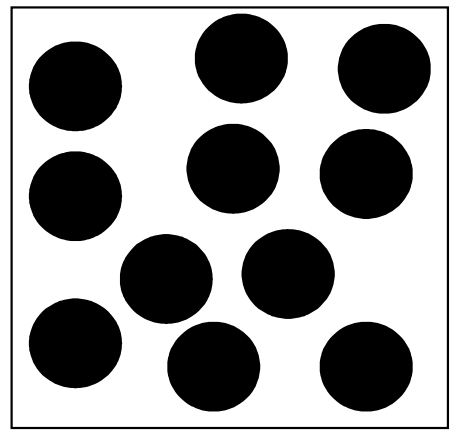

Figure 2. Sketch of hard-sphere matrix model.

In contrast to the overlapping HS model, the partition function obtained in Equation (49) is in fact independent of matrix configurations. Thus, we have the following:

$$
\Omega=\left\langle\Omega\left(\mathbf{q}^{N_{0}}\right)\right\rangle_{0}=\Omega\left(\mathbf{q}^{N_{0}}\right)=-k T \ln \Xi_{1}\left(\mathbf{q}^{N_{0}}\right)
$$

Then, one immediately finds the following expression for the thermodynamic pressure,

$$
P=\frac{-\Omega}{V}=k T z_{1}\left(1-\frac{\pi \sigma_{0}^{3} \rho_{0}}{6}\right)
$$

where

$$
\rho_{0}=\frac{1}{\Xi_{0}} \sum_{N_{0}=0}^{\infty} \frac{z_{0}^{N_{0}}}{\left(N_{0}-1\right) !} \int \mathrm{d} \mathbf{q}^{N_{0}-1} \mathrm{e}^{-\beta H_{00}}
$$

and

$$
\Xi_{0}=\sum_{N_{0}=0}^{\infty} \frac{z_{0}^{N_{0}}}{N_{0} !} \int \mathrm{d} \mathbf{q}^{N_{0}} \mathrm{e}^{-\beta H_{00}}
$$

are respectively the density and the partition function of the matrix.

The one-body distribution function for a particular matrix realization has exactly the same expression as that given in equation (31). Taking the average over matrix realizations, we find the following expression for the fluid density inside the matrix,

$$
\rho_{1}=\left\langle\rho_{1}^{(1)}\left(\mathbf{r} ; \mathbf{q}^{N_{0}}\right)\right\rangle_{0}=\frac{z_{1}}{\Xi_{0}} \sum_{N_{0}=0}^{\infty} \frac{z_{0}^{N_{0}}}{N_{0} !} \int \mathrm{d} \mathbf{q}^{N_{0}} \mathrm{e}^{-\beta H_{00}} \exp \left[-\beta \sum_{j=1}^{N_{0}} u_{10}\left(\left|\mathbf{r}-\mathbf{q}_{j}\right|\right)\right]
$$

At first glance, it does not seem possible to work out analytically the integral on the right hand side of the second equality of equation (54). However, it is worthwhile noting that the effect of

$$
\exp \left[-\beta \sum_{j=1}^{N_{0}} u_{10}\left(\left|\mathbf{r}-\mathbf{q}_{j}\right|\right)\right]
$$

in the integrand is just to exclude a spherical region (denoted by $V_{c}(\mathbf{r})$ ) with a radius equal to $\sigma_{0} / 2$ centered at $\mathbf{r}$ from the integration domain of each $\mathbf{q}_{j}$. Thus, equation (54) can be rewritten 
as follows:

$$
\begin{aligned}
\rho_{1}= & \frac{z_{1}}{\Xi_{0}} \sum_{N_{0}=0}^{\infty} \frac{z_{0}^{N_{0}}}{N_{0} !}\left(\int_{V} \mathrm{~d} \mathbf{q}_{1}-\int_{V_{c}(\mathbf{r})} \mathrm{d} \mathbf{q}_{1}\right)\left(\int_{V} \mathrm{~d} \mathbf{q}_{2}-\int_{V_{c}(\mathbf{r})} \mathrm{d} \mathbf{q}_{2}\right) \cdots\left(\int_{V} \mathrm{~d} \mathbf{q}_{N_{0}}-\int_{V_{c}(\mathbf{r})} \mathrm{d} \mathbf{q}_{N_{0}}\right) \mathrm{e}^{-\beta H_{00}} \\
= & \frac{z_{1}}{\Xi_{0}} \sum_{N_{0}=0}^{\infty} \frac{z_{0}^{N_{0}}}{N_{0} !}\left(\int_{V} \mathrm{~d} \mathbf{q}^{N_{0}}-N_{0} \int_{V_{c}(\mathbf{r})} \mathrm{d} \mathbf{q}_{1} \int_{V} \mathrm{~d} \mathbf{q}^{N_{0}-1}+\frac{N_{0}\left(N_{0}-1\right)}{2}\right. \\
& \left.\times \int_{V_{c}(\mathbf{r})} \mathrm{d} \mathbf{q}_{1} \int_{V_{c}(\mathbf{r})} \mathrm{d} \mathbf{q}_{2} \int_{V} \mathrm{~d} \mathbf{q}^{N_{0}-2}+\cdots\right) \mathrm{e}^{-\beta H_{00}} \\
= & z_{1}\left(1-\underset{V_{c}(\mathbf{r})}{\int} \mathrm{d} \mathbf{q}_{1} \rho_{0}+\frac{1}{2} \int_{V_{c}(\mathbf{r})} \mathrm{d} \mathbf{q}_{1} \int_{V_{c}(\mathbf{r})} \mathrm{d} \mathbf{q}_{2} \rho_{00}^{(2)}\left(\left|\mathbf{q}_{1}-\mathbf{q}_{2}\right|\right)+\cdots\right)=z_{1}\left(1-\frac{\pi \sigma_{0}^{3} \rho_{0}}{6}\right) .
\end{aligned}
$$

The expansion on the RHS of the third equality of equation (55) involves successively $\rho_{00}^{(n)}{ }_{0.0}\left(\mathbf{q}_{1}, \mathbf{q}_{2}, \ldots, \mathbf{q}_{n}\right)$. For the HS matrix model under consideration, all the terms containing $\rho_{00 \ldots 0}^{(n)}\left(\mathbf{q}_{1}, \mathbf{q}_{2}, \ldots, \mathbf{q}_{n}\right)$ with $n \geqslant 2$ have a vanishing contribution since the integration domain (which has the volume of $V_{c}(\mathbf{r})$ ) cannot contain two and more hard spheres of a diameter of $\sigma_{0}$. The Helmholtz free energy has the following expression in terms of $\rho_{1}$ and $\rho_{0}$,

$$
\frac{F}{V}=k T \rho_{1}\left[\ln \frac{\rho_{1} \Lambda_{1}^{3}}{1-\pi \sigma_{0}^{3} \rho_{0} / 6}-1\right]
$$

It should be noted that the Helmholtz free energy for this model cannot be expressed in a closed form in terms of $\mu_{1}$ and $\mu_{0}$.

The fluid-fluid two-body distribution function after averaging over matrix realizations is given by

$$
\begin{aligned}
\rho_{11}\left(\left|\mathbf{r}-\mathbf{r}^{\prime}\right|\right)= & \left\langle\rho_{1}^{(1)}\left(\mathbf{r} ; \mathbf{q}^{N_{0}}\right) \rho_{1}^{(1)}\left(\mathbf{r}^{\prime} ; \mathbf{q}^{N_{0}}\right)\right\rangle \\
= & \frac{z_{1}^{2}}{\Xi_{0}} \sum_{N_{0}=0}^{\infty} \frac{z_{0}^{N_{0}}}{N_{0} !} \int \mathrm{d} \mathbf{q}^{N_{0}} \mathrm{e}^{\beta H_{00}} \exp \left\{-\beta \sum_{j=1}^{N_{0}}\left[u_{10}\left(\left|\mathbf{r}-\mathbf{q}_{j}\right|\right)+u_{10}\left(\left|\mathbf{r}^{\prime}-\mathbf{q}_{j}\right|\right)\right]\right\} \\
= & \frac{z_{1}^{2}}{\Xi_{0}} \sum_{N_{0}=0}^{\infty} \frac{z_{0}^{N_{0}}}{N_{0} !}\left(\int_{V} \mathrm{~d} \mathbf{q}^{N_{0}}-N_{0} \int_{V_{d b}\left(\left|\mathbf{r}-\mathbf{r}^{\prime}\right|\right)} \mathrm{d} \mathbf{q}_{1} \int_{V} \mathrm{~d} \mathbf{q}^{N_{0}-1}\right. \\
& \left.+\frac{N_{0}\left(N_{0}-1\right)}{2} \int_{V_{d b}\left(\left|\mathbf{r}-\mathbf{r}^{\prime}\right|\right)} \mathrm{d} \mathbf{q}_{1} \int_{V_{d b}\left(\left|\mathbf{r}-\mathbf{r}^{\prime}\right|\right)} \mathrm{d} \mathbf{q}_{2} \int_{V} \mathrm{~d} \mathbf{q}^{N_{0}-2}+\cdots\right) \mathrm{e}^{-\beta H_{00}} \\
= & z_{1}^{2}\left(1-\rho_{0} V_{d b}\left(\left|\mathbf{r}-\mathbf{r}^{\prime}\right|\right)+\frac{1}{2} \int_{V_{d b}\left(\left|\mathbf{r}-\mathbf{r}^{\prime}\right|\right)} \mathrm{d} \mathbf{q}_{1} \int_{V_{d b}\left(\left|\mathbf{r}-\mathbf{r}^{\prime}\right|\right)} \mathrm{d} \mathbf{q}_{2} \rho_{00}^{(2)}\left(\mathbf{q}_{1}, \mathbf{q}_{2}\right)\right)
\end{aligned}
$$

where

$$
\rho_{00}^{(2)}\left(\mathbf{q}_{1}, \mathbf{q}_{2}\right)=\frac{1}{\Xi_{0}} \sum_{N_{0}=0}^{\infty} \frac{z_{0}^{N_{0}}}{\left(N_{0}-2\right) !} \int \mathrm{d} \mathbf{q}^{N_{0}-2} \mathrm{e}^{-\beta H_{00}},
$$


is the matrix-matrix two-body distribution function. In equation (57), a technique similar to the one used in equation (55) is used to remove

$$
\exp \left\{-\beta \sum_{j=1}^{N_{0}}\left[u_{10}\left(\left|\mathbf{r}-\mathbf{q}_{j}\right|\right)+u_{10}\left(\left|\mathbf{r}^{\prime}-\mathbf{q}_{j}\right|\right)\right]\right\}
$$

from the integrand by modifying the integration domains. In the present case, the exclusion region is a dumbbell with the two spheres centered at $\mathbf{r}$ and $\mathbf{r}^{\prime}$ with centered at $\mathbf{r}$ and $\mathbf{r}^{\prime}$ with a volume of $V_{d b}\left(\left|\mathbf{r}-\mathbf{r}^{\prime}\right|\right)$. The the term of three-body distribution function for the HS matrix model since the exclusion volume, $V_{d b}\left(\left|\mathbf{r}-\mathbf{r}^{\prime}\right|\right)$, cannot contain three and more hard spheres.

The matrix-fluid distribution function after averaging over matrix realizations is given by

$$
\begin{aligned}
& \rho_{01}(|\mathbf{q}-\mathbf{r}|)=\left\langle\rho_{1}^{(1)}\left(\mathbf{r} ; \mathbf{q}^{N_{0}}\right) \sum_{i=1}^{N_{0}} \delta\left(\mathbf{q}-\mathbf{q}_{i}\right)\right\rangle_{0} \\
&=\frac{z_{1}}{\Xi_{0}} \sum_{N_{0}=0}^{\infty} \frac{z_{0}^{N_{0}}}{N_{0} !} \int \mathrm{d} \mathbf{q}^{N_{0}} \mathrm{e}^{\beta H_{00}} \exp \left[-\beta \sum_{j=1}^{N_{0}} u_{10}\left(\left|\mathbf{r}-\mathbf{q}_{j}\right|\right)\right] \sum_{i=1}^{N_{0}} \delta\left(\mathbf{q}-\mathbf{q}_{i}\right) \\
&=\frac{z_{1} \exp \left[-\beta u_{10}(|\mathbf{r}-\mathbf{q}|)\right]}{\Xi_{0}} \sum_{N_{0}=0}^{\infty} \frac{z_{0}^{N_{0}}}{\left(N_{0}-1\right) !} \int \mathrm{d}_{\mathbf{q}^{N_{0}-1}} \mathrm{e}^{\beta H_{00}} \exp \left[-\beta \sum_{j=2}^{N_{0}} u_{10}\left(\left|\mathbf{r}-\mathbf{q}_{j}\right|\right)\right] \\
&\left.=\frac{z_{1} \exp \left[-\beta u_{10}(|\mathbf{r}-\mathbf{q}|)\right]}{\Xi_{0}} \sum_{N_{0}=0}^{\infty} \frac{z_{0}^{N_{0}}}{\left(N_{0}-1\right) !}\right] \\
& \quad \times\left[\int_{V} \mathrm{~d} \mathbf{q}^{N_{0}-1} \mathrm{e}^{\beta H_{00}}-\left(N_{0}-1\right) \int_{V_{c}(\mathbf{r})} \mathrm{d} \mathbf{q}_{2} \int_{V} \mathrm{~d} \mathbf{q}^{N_{0}-2} \mathrm{e}^{\beta H_{00}}+\cdots\right] \\
&= z_{1} \exp \left[-\beta u_{10}(|\mathbf{r}-\mathbf{q}|)\right] \\
& \rho_{0}-\int_{V_{c}(\mathbf{r})} d \mathbf{q}_{2} \rho_{00}^{(2)}\left(\mathbf{q}, \mathbf{q}_{2}\right)
\end{aligned}
$$

The expansion in the fourth equality of equation (59) truncates itself beyond the term of $\rho_{00}^{(2)}\left(\mathbf{q}, \mathbf{q}_{2}\right)$. For the HS matrix model, we cannot find entirely analytic expressions for different two-body distribution functions since we do not know the exact analytic expression of a HS fluid, $\rho_{00}^{(2)}\left(\mathbf{q}, \mathbf{q}^{\prime}\right)$.

\section{Sponge models}

\section{A. Random hard sponge model}

A sketch of the random hard sponge model is shown in figure 3. Recently, Zhao, Dong and Liu [31] have shown that the fluid-matrix interaction potential for this model can be described by

$$
H_{10}=\sum_{i=1}^{N_{1}} v_{10}\left(\mathbf{r}_{i} ; \mathbf{q}^{N_{0}}\right)
$$

where

$$
v_{10}\left(\mathbf{r}_{i} ; \mathbf{q}^{N_{0}}\right)=-k_{\mathrm{B}} T \ln \left[1-\mathrm{e}^{-\beta \sum_{j=1}^{N_{0}} u_{10}\left(\left|\mathbf{r}_{i}-\mathbf{q}_{j}\right|\right)}\right] .
$$


$u_{10}\left(\left|\mathbf{r}_{i}-\mathbf{q}_{j}\right|\right)$ in equation (61) is the potential given in equation (23). The other interaction potentials are specified by

$$
\begin{aligned}
& H_{00}=0, \\
& H_{11}=0 .
\end{aligned}
$$

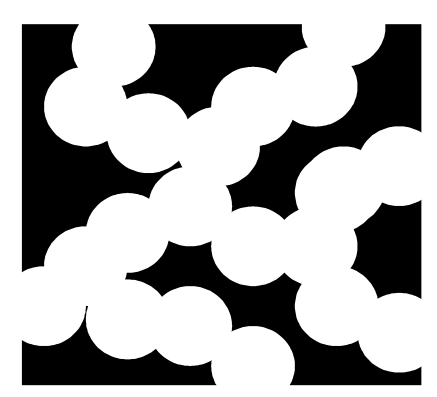

Figure 3. Sketch of the random hard sponge model.

The partition function for a particular matrix realization is given by

$$
\begin{aligned}
\Xi_{1}\left(\mathbf{q}^{N_{0}}\right) & =\exp \left\{z_{1} \int \mathrm{d} \mathbf{r}_{1} \exp \left[-\beta v_{10}\left(\mathbf{r}_{1} ; \mathbf{q}^{N_{0}}\right)\right]\right\} \\
& =\exp \left\{z_{1} \int \mathrm{d} \mathbf{r}_{1}\left[1-\exp \left[-\beta \sum_{j=1}^{N_{0}} u_{10}\left(\left|\mathbf{r}_{1}-\mathbf{q}_{j}\right|\right)\right]\right]\right\}
\end{aligned}
$$

It should be noted that the partition function in equation (64) can be rewritten as

$$
\Xi_{1}\left(\mathbf{q}^{N_{0}}\right)=\frac{\Xi_{1}^{\text {BulkIG }}}{\Xi_{1}^{\text {IG/OHSM }}\left(\mathbf{q}^{N_{0}}\right)}
$$

where

$$
\Xi_{1}^{\text {BulkIG }}=\mathrm{e}^{z_{1} V}
$$

is the partition function of a bulk ideal gas and $\Xi_{1}^{\mathrm{IG} / \mathrm{OHSM}}\left(\mathbf{q}^{N_{0}}\right)$ is the partition function of an ideal gas in a particular realization of an overlapping hard-sponge matrix [see equation (24)]. We obtain the following expression for the thermodynamic pressure,

$$
P=\frac{k T}{V}\left\langle\ln \Xi_{1}\left(\mathbf{q}^{N_{0}}\right)\right\rangle_{0}=k_{\mathrm{B}} T z_{1}\left[1-\exp \left(-\frac{\pi \sigma_{c}^{3} z_{0}}{6}\right)\right]=P^{\mathrm{BulkIG}}-P^{\mathrm{IG} / \mathrm{OHSM}}
$$

where

$$
P^{\text {BulkIG }}=k T z_{1}
$$

is the pressure of a bulk ideal gas and $P^{\mathrm{IG} / \mathrm{OHSM}}$ is the pressure of an ideal gas confined in an overlapping hard-sponge matrix (see equation (29) for its expression).

$$
\rho_{1}^{(1)}\left(\mathbf{r} ; \mathbf{q}^{N_{0}}\right)=z_{1} \exp \left[-\beta v_{10}\left(\mathbf{r} ; \mathbf{q}^{N_{0}}\right)\right]=\rho_{1}^{\text {BulkIG }}-\rho_{1}^{(1) \mathrm{IG} / \mathrm{OHSM}}\left(\mathbf{r} ; \mathbf{q}^{N_{0}}\right)
$$

where

$$
\rho_{1}^{\text {BulkIG }}=z_{1}
$$


is the density of an bulk ideal gas and $\rho_{1}^{(1) \mathrm{IG} / \mathrm{OHSM}}\left(\mathbf{r} ; \mathbf{q}^{N_{0}}\right)$ is the one-body distribution function of the fluid for a particular matrix realization given in equation (30). Taking the average over matrix realizations, we obtain

$$
\rho_{1}=z_{1}\left\langle\exp \left[-\beta v_{10}\left(\mathbf{r} ; \mathbf{q}^{N_{0}}\right)\right]\right\rangle_{0}=\rho_{1}^{\text {BulkIG }}-\rho_{1}^{\mathrm{IG} / \mathrm{OHSM}},
$$

where $\rho_{1}^{\mathrm{IG} / \mathrm{OHSM}}$ is the density of an ideal gas confined in the overlapping HS matrix whose expression is given in equation (31). The Helmholtz free energy has the following expression,

$$
\frac{F}{V}=\frac{F^{\mathrm{BulkIG}}}{V}-\frac{F^{\mathrm{IG} / \mathrm{OHSM}}}{V}
$$

where

$$
\frac{F^{\text {BulkIG }}}{V}=k T\left(\beta \mu_{1}-1\right) z_{1},
$$

is the Helmholtz free energy of a bulk ideal gas and $F^{\mathrm{IG} / \mathrm{OHSM}}$ is the Helmholtz free energy of an ideal gas confined in the overlapping HS matrix [see equation (33)]. In the above, we have seen that several thermodynamic functions, e.g., pressure, density and free energy, can be written as a contribution of a bulk ideal gas minus that of an ideal gas confined in the overlapping HS matrix [see equations (67), (71) and (72)]. Nevertheless, it should be pointed out that such decompositions hold only when these thermodynamic functions are expressed in terms of chemical potentials, i.e., $\mu_{1}$ and $\mu_{0}$. In terms of $\rho_{1}$ and $\rho_{0}$, we have

$$
P=k T \rho_{1}
$$

and

$$
\frac{F}{V}=k T \rho_{1}\left[\ln \frac{\rho_{1} \Lambda_{1}^{3}}{1-\mathrm{e}^{-\pi \sigma_{0}^{3} \rho_{0} / 6}}-1\right] .
$$

None of equations (74) and (75) has a form which can be decomposed into the contribution of a bulk ideal gas and that of an ideal gas confined in the overlapping HS matrix.

The fluid-fluid two-body distribution function averaged over matrix realizations is given by

$$
\begin{aligned}
\rho_{11}(\mid \mathbf{r} & \left.-\mathbf{r}^{\prime} \mid\right)=\left\langle\rho_{1}^{(1)}\left(\mathbf{r} ; \mathbf{q}^{N_{0}}\right) \rho_{1}^{(1)}\left(\mathbf{r}^{\prime} ; \mathbf{q}^{N_{0}}\right)\right\rangle=z_{1}^{2}-2 z_{1}\left\langle\rho_{1}^{(1) \mathrm{IG} / \mathrm{OHSM}}\left(\mathbf{r} ; \mathbf{q}^{N_{0}}\right)\right\rangle_{0} \\
& +\left\langle\rho_{1}^{(1) \mathrm{IG} / \mathrm{OHSM}}\left(\mathbf{r} ; \mathbf{q}^{N_{0}}\right) \rho_{1}^{(1) \mathrm{IG} / \mathrm{OHSM}}\left(\mathbf{r}^{\prime} ; \mathbf{q}^{N_{0}}\right)\right\rangle_{0} \\
= & z_{1}^{2}-2 z_{1} \rho_{1}^{\mathrm{IG} / \mathrm{OHSM}}+\rho_{11}^{\mathrm{IG} / \mathrm{OHSM}}\left(\left|\mathbf{r}-\mathbf{r}^{\prime}\right|\right)=z_{1}^{2}\left[1-2 \mathrm{e}^{-\pi \sigma_{0}^{3} z_{0} / 6}+\mathrm{e}^{\left.-z_{0} V_{d b}\left(\left|\mathbf{r}-\mathbf{r}^{\prime}\right|\right)\right],}\right.
\end{aligned}
$$

where $V_{d b}\left(\left|\mathbf{r}-\mathbf{r}^{\prime}\right|\right)$ was given in equation (36).

The fluid-matrix two-body distribution function averaged over matrix configurations has the following expression,

$$
\begin{aligned}
\rho_{01}(|\mathbf{q}-\mathbf{r}|) & =\left\langle\rho_{1}^{(1)}\left(\mathbf{r} ; \mathbf{q}^{N_{0}}\right) \sum_{i=1}^{N_{0}} \delta\left(\mathbf{q}-\mathbf{q}_{i}\right)\right\rangle_{0} \\
& =\left\langle\rho_{1}^{\text {BulkIG }} \sum_{i=1}^{N_{0}} \delta\left(\mathbf{q}-\mathbf{q}_{i}\right)\right\rangle_{0}-\left\langle\rho_{1}^{(1) I G / O H S M}\left(\mathbf{r} ; \mathbf{q}^{N_{0}}\right) \sum_{i=1}^{N_{0}} \delta\left(\mathbf{q}-\mathbf{q}_{i}\right)\right\rangle_{0} \\
& =\rho_{1}^{\text {BulkIG }} \rho_{0}^{\text {BulkIG }}-\rho_{01}^{\text {IG } / \text { OHSM }}(|\mathbf{q}-\mathbf{r}|)=z_{0} z_{1}\left[1-\mathrm{e}^{-\pi \sigma_{0}^{3} z_{0} / 6} \mathrm{e}^{-\beta u_{01}(|\mathbf{q}-\mathbf{r}|)}\right] .
\end{aligned}
$$




\section{B. Soft-sponge model}

Recently, Dong, Krakoviack and Zhao have proposed a soft sponge model [32]. A sketch of this model is presented in figure 4.

We consider here the simple case of a soft-sponge matrix with totally randomly distributed cavities. The fluid-matrix interaction potential is

$$
H_{10}=\varepsilon \sum_{i=1}^{N_{1}} \theta\left(\mathbf{r}_{i} ; \mathbf{q}^{N_{0}}\right),
$$

where the generalized Heaviside function is given by

$$
\theta\left(\mathbf{r} ; \mathbf{q}^{N_{0}}\right)=\mathrm{e}^{-\beta \sum_{j=1}^{N_{0}} u_{10}\left(\left|\mathbf{r}-\mathbf{q}_{j}\right|\right)} .
$$

$u_{10}(|\mathbf{r}-\mathbf{q}|)$ in equation (79) is the potential given in equation (23). The partition function in the grand canonical ensemble for a particular matrix realization is given by

$$
\Xi_{1}\left(\mathbf{q}^{N_{0}}\right)=\exp \left[z_{1} \int \mathrm{d} \mathbf{r}_{1} \mathrm{e}^{-\beta \varepsilon \theta\left(\mathbf{r}_{1} ; \mathbf{q}^{N_{0}}\right)}\right] .
$$

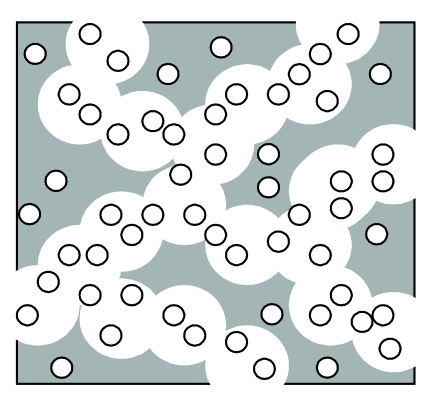

Figure 4. Sketch of soft sponge matrix with a solid medium (represented in grey) permeable to fluid particles (small circles).

The grand partition function for a particular matrix realization is

$$
\Omega_{1}\left(\mathbf{q}^{N_{0}}\right)=-k T \ln \Xi_{1}\left(\mathbf{q}^{N_{0}}\right)=-k T z_{1} \int \mathrm{d} \mathbf{r}_{1} \mathrm{e}^{-\beta \varepsilon \theta\left(\mathbf{r}_{1} ; \mathbf{q}^{N_{0}}\right)}
$$

Averaging over matrix realizations, we have

$$
\left.\Omega=\left\langle\Omega_{1}\left(\mathbf{q}^{N_{0}}\right)\right\rangle_{0}=-k T z_{1} \int \mathrm{d} \mathbf{r}_{1}\left\langle\mathrm{e}^{-\beta \varepsilon \theta\left(\mathbf{r}_{1} ; \mathbf{q}^{N_{0}}\right.}\right)\right\rangle_{0}
$$

To be able to carry out the average in equation $(82)$, we rewrite $\mathrm{e}^{-\beta \varepsilon \theta\left(\mathbf{r}_{1} ; \mathbf{q}^{N_{0}}\right)}$ in the following form,

$$
\begin{aligned}
\mathrm{e}^{-\beta \varepsilon \theta\left(\mathbf{r}_{1} ; \mathbf{q}^{N_{0}}\right)} & =1-\beta \varepsilon \theta\left(\mathbf{r}_{1} ; \mathbf{q}^{N_{0}}\right)+\frac{1}{2}\left[\beta \varepsilon \theta\left(\mathbf{r}_{1} ; \mathbf{q}^{N_{0}}\right)\right]^{2}-\frac{1}{3 !}\left[\beta \varepsilon \theta\left(\mathbf{r}_{1} ; \mathbf{q}^{N_{0}}\right)\right]^{3}+\cdots \\
& =1+\theta\left(\mathbf{r}_{1} ; \mathbf{q}^{N_{0}}\right)\left[-\beta \varepsilon+\frac{1}{2}(\beta \varepsilon)^{2}-\frac{1}{3 !}(\beta \varepsilon)^{3}+\cdots\right] \\
& =1+\left(\mathrm{e}^{-\beta \varepsilon}-1\right) \theta\left(\mathbf{r}_{1} ; \mathbf{q}^{N_{0}}\right)
\end{aligned}
$$


When going to the second equality of equation (83), the following property of Heaviside function was used,

$$
\left[\theta\left(\mathbf{r}_{1} ; \mathbf{q}^{N_{0}}\right)\right]^{n}=\theta\left(\mathbf{r}_{1} ; \mathbf{q}^{N_{0}}\right), \quad(n=1,2,3, \ldots)
$$

Substituting equation (83) into equation (82), we obtain

$$
\Omega=-k T z_{1}\left[V+\left(\mathrm{e}^{-\beta \varepsilon}-1\right) \int \mathrm{d} \mathbf{r}_{1}\left\langle\theta\left(\mathbf{r}_{1} ; \mathbf{q}^{N_{0}}\right)\right\rangle_{0}\right] .
$$

The average of $\theta\left(\mathbf{r}_{1} ; \mathbf{q}^{N_{0}}\right)$ over matrix realizations can be carried out analytically in the case of totally randomly distributed cavities.

$$
\begin{aligned}
\left\langle\theta\left(\mathbf{r}_{1} ; \mathbf{q}^{N_{0}}\right)\right\rangle_{0} & =\frac{1}{\Xi_{0}} \sum_{N_{0}=0}^{\infty} \frac{z_{0}^{N_{0}}}{N_{0} !} \int \mathrm{d} \mathbf{q}^{N_{0}} \mathrm{e}^{-\beta \sum_{j=1}^{N_{0}} \phi_{10}\left(\left|\mathbf{r}_{1}-\mathbf{q}_{j}\right|\right)} \\
& =\frac{1}{\Xi_{0}} \sum_{N_{0}=0}^{\infty} \frac{z_{0}^{N_{0}}}{N_{0} !}\left(V-\frac{\pi \sigma_{10}^{3}}{6}\right)^{N_{0}}=\mathrm{e}^{-\frac{\pi \sigma_{10}^{3} z_{0}}{6}} .
\end{aligned}
$$

Substituting equation (86) into equation (85), we obtain

$$
\Omega=-k T z_{1} V\left[1+\left(\mathrm{e}^{-\beta \varepsilon}-1\right) \mathrm{e}^{-\frac{\pi \sigma_{10}^{3} z_{0}}{6}}\right] .
$$

Then, we immediately obtain the following result for pressure,

$$
P=-\frac{\Omega}{V}=k T z_{1}\left[1+\left(\mathrm{e}^{-\beta \varepsilon}-1\right) \mathrm{e}^{-\frac{\pi \sigma_{10}^{3} z_{0}}{6}}\right] .
$$

The one-body distribution function for a particular matrix realization is given by

$$
\rho_{1}^{(1)}\left(\mathbf{r} ; \mathbf{q}^{N_{0}}\right)=z_{1} \mathrm{e}^{-\beta \varepsilon \theta\left(\mathbf{r} ; \mathbf{q}^{N_{0}}\right)}=z_{1}\left[1+\left(\mathrm{e}^{-\beta \varepsilon}-1\right) \theta\left(\mathbf{r}_{1} ; \mathbf{q}^{N_{0}}\right)\right] .
$$

The averaging over matrix realizations leads to

$$
\rho_{1}=\left\langle\rho_{1}^{(1)}\left(\mathbf{r} ; \mathbf{q}^{N_{0}}\right)\right\rangle_{0}=z_{1}\left[1+\left(\mathrm{e}^{-\beta \varepsilon}-1\right) \mathrm{e}^{-\frac{\pi \sigma_{10}^{3} z_{0}}{6}}\right] .
$$

The Helmholtz free energy has the following expressions,

$$
\frac{F}{V}=k T\left(\beta \mu_{1}-1\right) z_{1}\left[1+\left(\mathrm{e}^{-\beta \varepsilon}-1\right) \mathrm{e}^{-\frac{\pi \sigma_{10}^{3} z_{0}}{6}}\right],
$$

and

$$
\frac{F}{V}=k T \rho_{1}\left[\ln \frac{\rho_{1} \Lambda_{1}^{3}}{1+\left(\mathrm{e}^{-\beta \varepsilon}-1\right) \mathrm{e}^{-\pi \sigma_{10}^{3} z_{0} / 6}}-1\right] .
$$

Unlike in the hard sponge model, the mean potential energy in the soft sponge model is non-zero and is given by

$$
\begin{aligned}
E & =\left\langle\left\langle H_{10}\right\rangle_{1}\right\rangle_{0}=\left\langle\frac{1}{\Xi_{1}\left(\mathbf{q}^{N_{0}}\right)} \sum_{N_{1}=0}^{\infty} \frac{z_{1}^{N_{1}}}{N_{1} !} \int \mathrm{d} \mathbf{r}^{N_{1}} H_{10} e^{-\beta H_{10}}\right\rangle_{0}=\left\langle\varepsilon \int \mathrm{d} \mathbf{r}_{1} \theta\left(\mathbf{r}_{1} ; \mathbf{q}^{N_{0}}\right) \rho_{1}^{(1)}\left(\mathbf{r}_{1} ; \mathbf{q}^{N_{0}}\right)\right\rangle_{0} \\
& =\varepsilon_{1}^{z} \mathrm{e}^{-\beta \varepsilon} \int \mathrm{d} \mathbf{r}_{1}\left\langle\theta\left(\mathbf{r}_{1} ; \mathbf{q}^{N_{0}}\right)\right\rangle_{0}=\varepsilon_{V} z_{1} \mathrm{e}^{-\beta \varepsilon} \mathrm{e}^{-\frac{\pi \sigma_{10}^{3} z_{0}}{6}}
\end{aligned}
$$


The fluid-fluid two-body distribution function is given by

$$
\begin{aligned}
\rho_{11}^{(2)}\left(\left|\mathbf{r}-\mathbf{r}^{\prime}\right|\right)= & \left\langle\rho_{1}^{(1)}\left(\mathbf{r} ; \mathbf{q}^{N_{0}}\right) \rho_{1}^{(1)}\left(\mathbf{r}^{\prime} ; \mathbf{q}^{N_{0}}\right)\right\rangle_{0} \\
= & z_{1}^{2}\left\langle 1+\left(\mathrm{e}^{-\beta \varepsilon}-1\right) \theta\left(\mathbf{r} ; \mathbf{q}^{N_{0}}\right)+\left(\mathrm{e}^{-\beta \varepsilon}-1\right) \theta\left(\mathbf{r}^{\prime} ; \mathbf{q}^{N_{0}}\right)\right. \\
& \left.\quad+\left(\mathrm{e}^{-\beta \varepsilon}-1\right)^{2} \theta\left(\mathbf{r} ; \mathbf{q}^{N_{0}}\right) \theta\left(\mathbf{r}^{\prime} ; \mathbf{q}^{N_{0}}\right)\right\rangle_{0} \\
= & z_{1}^{2}\left[1+2\left(\mathrm{e}^{-\beta \varepsilon}-1\right)\left\langle\theta\left(\mathbf{r} ; \mathbf{q}^{N_{0}}\right)\right\rangle_{0}+\left(\mathrm{e}^{-\beta \varepsilon}-1\right)^{2}\left\langle\theta\left(\mathbf{r} ; \mathbf{q}^{N_{0}}\right) \theta\left(\mathbf{r}^{\prime} ; \mathbf{q}^{N_{0}}\right)\right\rangle_{0}\right] \\
= & z_{1}^{2}\left[1+2\left(\mathrm{e}^{-\beta \varepsilon}-1\right) \mathrm{e}^{-\frac{\pi \sigma_{10}^{3} z_{0}}{6}}+\left(\mathrm{e}^{-\beta \varepsilon}-1\right)^{2} \mathrm{e}^{\left.-z_{0} V_{d b}\left(\left|\mathbf{r}-\mathbf{r}^{\prime}\right|\right)\right],}\right.
\end{aligned}
$$

where $V_{d b}\left(\left|\mathbf{r}-\mathbf{r}^{\prime}\right|\right)$ is the volume of a dumbbell whose expression is already volume of a dumbbell whose expression is already given in equation (36). The result for the matrix-fluid two-body distribution function is

$$
\begin{aligned}
\rho_{01}^{(2)}(|\mathbf{r}-\mathbf{q}|) & =\left\langle\rho_{1}^{(1)}\left(\mathbf{r} ; \mathbf{q}^{N_{0}}\right) \sum_{j=1}^{N_{0}} \delta\left(\mathbf{q}_{j}-\mathbf{q}\right)\right\rangle_{0} \\
& =\left\langle z_{1}\left[1+\left(\mathrm{e}^{-\beta \varepsilon}-1\right) \theta\left(\mathbf{r}_{1} ; \mathbf{q}^{N_{0}}\right)\right] \sum_{j=1}^{N_{0}} \delta\left(\mathbf{q}_{j}-\mathbf{q}\right)\right\rangle_{0} \\
& =z_{1}\left[\rho_{0}+\left(\mathrm{e}^{-\beta \varepsilon}-1\right) z_{0} \mathrm{e}^{-\frac{\pi \sigma_{10}^{3} z_{0}}{6}} \mathrm{e}^{-\beta \phi_{01}(|\mathbf{q}-\mathbf{r}|)}\right]
\end{aligned}
$$

It is straight to check that all the above results reduce to those of the hard-sponge model, i.e., the results given in the last subsection.

\section{Models of templated matrices}

\section{A. Zhao-Dong-Liu model}

The sketch of the templated matrix model proposed by Zhao, Dong and Liu recently [33] is given in figure 5. Starting with a primitive matrix (e.g., a Madden-Glandt matrix), one builds the templated matrix by piercing some spherical cavities in the primitive one (see figure 5).

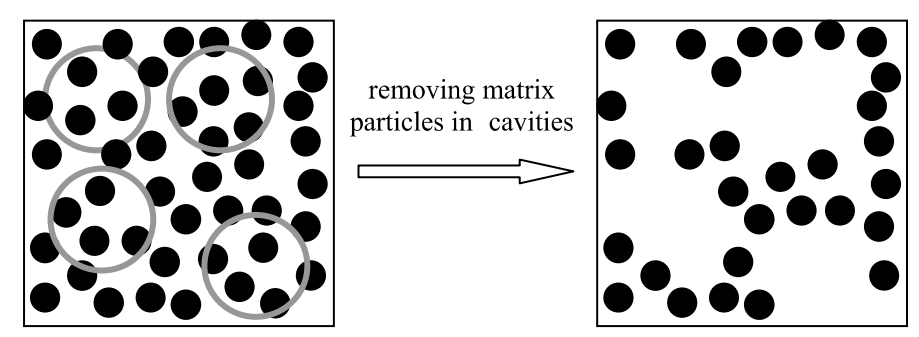

Figure 5. Sketch of Zhao-Dong-Liu model of templated matrices: matrix particles represented by black spheres and cavities represented by grey empty spheres.

Here, we consider the simplest case of the above templated matrix in which the matrix particles and the cavities are distributed completely randomly, i.e.,

$$
H_{00}=0
$$

and

$$
H_{0^{\prime} 0^{\prime}}=0
$$


where the index 0 denotes the matrix species as before and the index $0^{\prime}$ denotes the cavity species. We denote this templated matrix as RHS/RC matrix (random HS with random cavities). The fluid-matrix interaction potentials is given by

$$
H_{10}=\sum_{i=1}^{N_{1}} \sum_{j=1}^{N_{0}} u_{10}\left(\left|\mathbf{r}_{\mathrm{i}}-\mathbf{q}_{\mathbf{j}}\right|\right) \theta\left(\mathbf{q}_{j} ; \mathbf{Q}^{N_{0^{\prime}}}\right),
$$

where

$$
\theta\left(\mathbf{q}_{j} ; \mathbf{Q}^{N_{0^{\prime}}}\right)=\exp \left[-\beta \sum_{k=0}^{N_{0^{\prime}}} u_{00^{\prime}}\left(\left|\mathbf{q}_{j}-\mathbf{Q}_{k}\right|\right)\right],
$$

is a generalized Heaviside function which is equal to 1 when $\mathbf{q}_{j}$ is outside a cavity and to 0 when $\mathbf{q}_{j}$ is inside a cavity $\left(\mathbf{Q}_{k}\right.$ specifies the position of the center of the k-th equation (23) and $u_{00^{\prime}}$ is given by

$$
u_{00^{\prime}}\left(\left|\mathbf{q}_{j}-\mathbf{Q}_{k}\right|\right)=\left\{\begin{array}{cc}
\infty, & \left|\mathbf{q}_{j}-\mathbf{Q}_{k}\right|<\sigma_{0^{\prime}} / 2 \\
0, & \left|\mathbf{q}_{j}-\mathbf{Q}_{k}\right| \geqslant \sigma_{0^{\prime}} / 2
\end{array}\right.
$$

where $\sigma_{0^{\prime}}$ is obviously the cavity diameter.

First, we describe some quantities characterizing the empty matrix. By the construction of the model, some matrix particles are removed (those falling in cavities). Thus, the matrix particle number fluctuates in different matrix and cavity realizations. The matrix particle density for a particular cavity realization is given by

$$
\begin{aligned}
\rho_{0}^{(1)}\left(\mathbf{q} ; \mathbf{Q}^{N_{0^{\prime}}}\right) & =\left\langle\sum_{i=1}^{N_{0}} \delta\left(\mathbf{q}_{i}-\mathbf{q}\right) \theta\left(\mathbf{q}_{i} ; \mathbf{Q}^{N_{0^{\prime}}}\right)\right\rangle_{0}=\frac{1}{\Xi_{0}} \sum_{N_{0}=0}^{\infty} \frac{z_{0}^{N_{0}}}{N_{0} !} \int \mathrm{d} \mathbf{q}^{N_{0}} \sum_{i=1}^{N_{0}} \delta\left(\mathbf{q}_{i}-\mathbf{q}\right) \theta\left(\mathbf{q}_{i} ; \mathbf{Q}^{N_{0^{\prime}}}\right) \\
& =\frac{\theta\left(\mathbf{q} ; \mathbf{Q}^{N_{0^{\prime}}}\right)}{\Xi_{0}} \sum_{N_{0}=0}^{\infty} \frac{z_{0}^{N_{0}}}{\left(N_{0}-1\right) !} V^{N_{0}-1}=z_{0} \theta\left(\mathbf{q} ; \mathbf{Q}^{N_{0^{\prime}}}\right)=\tilde{\rho}_{0} \theta\left(\mathbf{q} ; \mathbf{Q}^{N_{0^{\prime}}}\right),
\end{aligned}
$$

where $\tilde{\rho}_{0}$ is the density of the primitive matrix. In the definition of $\rho_{0}^{(1)}\left(\mathbf{q} ; \mathbf{Q}^{N_{0^{\prime}}}\right)$ given in equation (101), we must multiply $\delta\left(\mathbf{q}_{i}-\mathbf{q}\right)$ by $\theta\left(\mathbf{q}_{i} ; \mathbf{Q}^{N_{0^{\prime}}}\right)$ so that the matrix particles falling in a cavity are not counted in the calculation of matrix density. Equation (101) clearly shows that the one-body matrix distribution function in the templated matrix for a particular cavity configuration is equal to zero inside the cavities and to $\tilde{\rho}_{0}$ elsewhere, which is precisely what one can anticipate for this model. We obtain the mean matrix density by taking the average of $\rho_{0}^{(1)}\left(\mathbf{q} ; \mathbf{Q}^{N_{0^{\prime}}}\right)_{0}$ over cavity realizations,

$$
\rho_{0}=\left\langle\rho_{0}^{(1)}\left(\mathbf{q} ; \mathbf{Q}^{N_{0^{\prime}}}\right)\right\rangle_{0^{\prime}}=\tilde{\rho}_{0}\left\langle\theta\left(\mathbf{q} ; \mathbf{Q}^{N_{0^{\prime}}}\right)\right\rangle_{0^{\prime}}
$$

where

$$
\begin{aligned}
\left\langle\theta\left(\mathbf{q} ; \mathbf{Q}^{N_{0^{\prime}}}\right)\right\rangle_{0^{\prime}} & =\frac{1}{\Xi_{0^{\prime}}} \sum_{N_{0^{\prime}}=0}^{\infty} \frac{z_{0^{\prime}}^{N_{0^{\prime}}}}{N_{0^{\prime}} !} \int \mathrm{d} \mathbf{Q}^{N_{0^{\prime}}} \exp \left[-\beta \sum_{j=1}^{N_{0^{\prime}}} u_{00^{\prime}}\left(\left|\mathbf{q}-\mathbf{Q}_{j}\right|\right)\right] \\
& =\frac{1}{\Xi_{0^{\prime}}} \sum_{N_{0^{\prime}}=0}^{\infty} \frac{z_{0^{\prime}}^{N_{0^{\prime}}}}{N_{0^{\prime}} !}\left(V-\frac{\pi \sigma_{0^{\prime}}^{3}}{6}\right)^{N_{0^{\prime}}}=\mathrm{e}^{-\pi \sigma_{0^{\prime}}^{3}, z_{0^{\prime}} / 6} .
\end{aligned}
$$

Substituting equation (103) into equation (102), we finally obtain,

$$
\rho_{0}=\tilde{\rho}_{0} \mathrm{e}^{-\pi \sigma_{0^{\prime}}^{3} \rho_{0^{\prime}} / 6} .
$$

The result given by equation (104) has an appealing physical meaning: the average matrix particle density is given by the product of the density of the primitive matrix and the probability 
of a matrix particle not falling into a cavity. It should also be noted that the density of RHS/RC matrix is given by the same expression as the one for the fluid density in a RHS matrix [compare equation (104) with equation (31)].

Then we calculate the matrix-matrix two-body distribution function. For a particular cavity realization, it is given by

$$
\begin{aligned}
\rho_{00}^{(2)}\left(\mathbf{q}, \mathbf{q}^{\prime} ; \mathbf{Q}^{N_{0}}\right) & =\left\langle\sum_{i=1}^{N_{0}} \sum_{j=1, j \neq i}^{N_{0}} \delta\left(\mathbf{q}_{i}-\mathbf{q}\right) \delta\left(\mathbf{q}_{j}-\mathbf{q}^{\prime}\right) \theta\left(\mathbf{q}_{i} ; \mathbf{Q}^{N_{0}}\right) \theta\left(\mathbf{q}_{j} ; \mathbf{Q}^{N_{0}}\right)\right\rangle_{0} \\
& =\frac{1}{\Xi} \sum_{N_{0}=0}^{\infty} \frac{z_{0}^{N_{0}}}{N_{0} !} \int \mathrm{d} \mathbf{q}^{N_{0}} \sum_{i=1}^{N_{0}} \sum_{j=1, j \neq i}^{N_{0}} \delta\left(\mathbf{q}_{i}-\mathbf{q}\right) \delta\left(\mathbf{q}_{j}-\mathbf{q}^{\prime}\right) \theta\left(\mathbf{q}_{i} ; \mathbf{Q}^{N_{0^{\prime}}}\right) \theta\left(\mathbf{q}_{j} ; \mathbf{Q}^{N_{0}}\right) \\
& =\frac{\theta\left(\mathbf{q} ; \mathbf{Q}^{N_{0^{\prime}}}\right) \theta\left(\mathbf{q}^{\prime} ; \mathbf{Q}^{N_{0^{\prime}}}\right)}{\Xi_{0}} \sum_{N_{0}=0}^{\infty} \frac{z_{0}^{N_{0}}}{\left(N_{0}-2\right) !} V^{N_{0}-2}=z_{0}^{2} \theta\left(\mathbf{q} \mathbf{Q}^{N_{0^{\prime}}}\right) \theta\left(\mathbf{q}^{\prime} ; \mathbf{Q}^{N_{0}}\right) \\
& =\tilde{\rho}_{0}^{2} \theta\left(\mathbf{q} ; \mathbf{Q}^{N_{0^{\prime}}}\right)=\rho_{0}^{(1)}\left(\mathbf{q} ; \mathbf{Q}^{N_{0^{\prime}}}\right) \rho_{0}^{(1)}\left(\mathbf{q}^{\prime} ; \mathbf{Q}^{N_{0}^{\prime}}\right) .
\end{aligned}
$$

The averaging over cavity realizations leads to

$$
\rho_{00}^{(2)}\left(\left|\mathbf{q}-\mathbf{q}^{\prime}\right|\right)=\left\langle\rho_{00}^{(2)}\left(\mathbf{q}, \mathbf{q}^{\prime} ; \mathbf{Q}^{N_{0^{\prime}}}\right)\right\rangle_{0}=\tilde{\rho}_{0}^{2}\left\langle\theta\left(\mathbf{q} ; \mathbf{Q}^{N_{0^{\prime}}}\right) \theta\left(\mathbf{q}^{\prime} ; \mathbf{Q}^{N_{0^{\prime}}}\right)\right\rangle_{0^{\prime}},
$$

and

$$
\begin{aligned}
\left\langle\theta\left(\mathbf{q} ; \mathbf{Q}^{N_{0}^{\prime}}\right) \theta\left(\mathbf{q}^{\prime} ; \mathbf{Q}^{\mathbf{N}_{\mathbf{o}}^{\prime}}\right)\right\rangle_{0^{\prime}} & =\frac{1}{\Xi} \sum_{N_{0^{\prime}}=0}^{\infty} \frac{z_{0^{\prime}}^{N_{0^{\prime}}}}{N_{0} !} \int \mathrm{d} \mathbf{Q}^{N_{0^{\prime}}} \exp \left\{-\beta \sum_{j=0}^{N_{0^{\prime}}}\left[u_{00^{\prime}}\left(\left|\mathbf{q}-\mathbf{Q}_{j}\right|+u_{00^{\prime}}\left|\mathbf{q}^{\prime}-\mathbf{Q}_{j}\right|\right)\right]\right\} \\
& =\frac{1}{\Xi} \sum_{0^{\prime}}^{\infty} \frac{z_{0^{\prime}}^{N_{0}^{\prime}}}{N_{0^{\prime}} !}\left(V-V_{d b}\left(\left|\mathbf{q}-\mathbf{q}^{\prime}\right|\right)\right)^{N_{0^{\prime}}}=\mathrm{e}^{-z_{0^{\prime}} V_{d b}\left(\left|\mathbf{q}-\mathbf{q}^{\prime}\right|\right)} \\
& =\mathrm{e}^{-\rho_{0^{\prime}} V_{d b}\left(\left|\mathbf{q}-\mathbf{q}^{\prime}\right|\right)}
\end{aligned}
$$

where $V_{d b}\left(\left|\mathbf{q}-\mathbf{q}^{\prime}\right|\right)$ is the volume of a dumbbell formed by two spheres with a diameter of $\sigma_{0^{\prime}}$ located at $\mathbf{q}$ and $\mathbf{q}^{\prime}$ (see equation (36) for its expression). Substituting expression). Substituting equation (107) into equation (106), we obtain

$$
\rho_{00}^{(2)}\left(\left|\mathbf{q}-\mathbf{q}^{\prime}\right|\right)=\tilde{\rho}_{0}^{2} e^{-\rho_{0^{\prime}} V_{d b}\left(\left|\mathbf{q}-\mathbf{q}^{\prime}\right|\right)} .
$$

The above result has also a very appealing physical meaning. $\rho_{00}^{(2)}\left(\left|\mathbf{q}-\mathbf{q}^{\prime}\right|\right)$ is given by the twobody distribution of the primitive matrix, i.e., $\tilde{\rho}_{0}^{2}$, multiplied by the probability that none of the two points (at $\mathbf{q}$ and $\mathbf{q}^{\prime}$ ) falls into a cavity.

The matrix-cavity two-body distribution function for a particular cavity realization is given by

$$
\begin{aligned}
\rho_{00^{\prime}}^{(2)}\left(\mathbf{q}, \mathbf{Q} ; \mathbf{Q}^{N_{0^{\prime}}}\right) & =\left\langle\sum_{i=1}^{N_{0}} \sum_{j=1}^{N_{0^{\prime}}} \delta\left(\mathbf{q}_{i}-\mathbf{q}\right) \theta\left(\mathbf{q}_{i} ; \mathbf{Q}^{N_{0^{\prime}}}\right) \delta\left(\mathbf{Q}_{j}-\mathbf{Q}\right)\right\rangle_{0} \\
& =\left\langle\sum_{i=1}^{N_{0}} \delta\left(\mathbf{q}_{i}-\mathbf{q}\right) \theta\left(\mathbf{q}_{i} ; \mathbf{Q}^{N_{0^{\prime}}}\right)\right\rangle_{0} \sum_{j=1}^{N_{0^{\prime}}} \delta\left(\mathbf{Q}_{j}-\mathbf{Q}\right) \\
& =\rho_{0}^{(1)}\left(\mathbf{q} ; \mathbf{Q}^{N_{0^{\prime}}}\right) \sum_{j=1}^{N_{0^{\prime}}} \delta\left(\mathbf{Q}_{j}-\mathbf{Q}\right)=\tilde{\rho}_{0} \theta\left(\mathbf{q} ; \mathbf{Q}^{N_{0^{\prime}}}\right) \sum_{j=1}^{N_{0^{\prime}}} \delta\left(\mathbf{Q}_{j}-\mathbf{Q}\right) .
\end{aligned}
$$

Taking the average over cavity realizations, we obtain

$$
\rho_{00^{\prime}}^{(2)}(|\mathbf{q}-\mathbf{Q}|)=\tilde{\rho}_{0}\left\langle\theta\left(\mathbf{q} ; \mathbf{Q}^{N_{0^{\prime}}}\right) \sum_{j=1}^{N_{0^{\prime}}} \delta\left(\mathbf{Q}_{\mathbf{j}}-\mathbf{Q}\right)\right\rangle_{0}
$$


where

$$
\begin{gathered}
\left\langle\theta\left(\mathbf{q} ; \mathbf{Q}^{N_{0^{\prime}}}\right) \sum_{j=1}^{N_{0^{\prime}}} \delta\left(\mathbf{Q}_{\mathbf{j}}-\mathbf{Q}\right)\right\rangle=\frac{1}{\Xi_{0^{\prime}}} \sum_{N_{0^{\prime}}=0}^{\infty} \frac{z_{0^{\prime}}^{N_{0^{\prime}}}}{N_{0^{\prime}} !} \int \mathrm{d} \mathbf{Q}^{N_{0^{\prime}}} \sum_{j=1}^{N_{0^{\prime}}} \delta\left(\mathbf{Q}_{j}-\mathbf{Q}\right) \exp \left[-\beta \sum_{i=1}^{N_{0^{\prime}}} u_{00^{\prime}}\left(\left|\mathbf{q}-\mathbf{Q}_{i}\right|\right)\right] \\
=\frac{\mathrm{e}^{-\beta u_{00^{\prime}}(|\mathbf{q}-\mathbf{Q}|)}}{\Xi_{0}} \sum_{N_{0^{\prime}}=0}^{\infty} \frac{z_{0^{\prime}}^{N_{0^{\prime}}}}{\left(N_{0^{\prime}}-1\right) !} \int \mathrm{d} \mathbf{Q}^{N_{0^{\prime}}-1} \exp \left[-\beta \sum_{i=2}^{N_{0^{\prime}}} u_{00^{\prime}}\left(\left|\mathbf{q}-\mathbf{Q}_{i}\right|\right)\right] \\
=\frac{\mathrm{e}^{-\beta u_{00^{\prime}}(|\mathbf{q}-\mathbf{Q}|)}}{\Xi_{0}} \sum_{N_{0^{\prime}}=0}^{\infty} \frac{z_{0^{\prime}}^{N_{0^{\prime}}}}{\left(N_{0^{\prime}}-1\right) !}\left(V-\frac{\pi \sigma_{0^{\prime}}^{3}}{\sigma}\right)^{N_{0^{\prime}}-1} \\
=z_{0^{\prime}} \mathrm{e}^{-\beta u_{00^{\prime}}(|\mathbf{q}-\mathbf{Q}|)} \mathrm{e}^{-\pi \sigma_{o^{\prime}}^{3} z_{0^{\prime}} / 6}=\rho_{0^{\prime}} \mathrm{e}^{-\beta u_{00^{\prime}}(|\mathbf{q}-\mathbf{Q}|)} \mathrm{e}^{-\pi \sigma_{o^{\prime}}^{3} \rho_{0^{\prime}} / 6} .
\end{gathered}
$$

We finally find

$$
\rho_{00^{\prime}}^{(2)}(|\mathbf{q}-\mathbf{Q}|)=\tilde{\rho}_{0} \rho_{0^{\prime}} \mathrm{e}^{-\beta u_{00^{\prime}}(|\mathbf{q}-\mathbf{Q}|)} \mathrm{e}^{-\pi \sigma_{0^{\prime}}^{3} \rho_{0^{\prime}} / 6}
$$

Now, we proceed to the calculation of various properties when an ideal is adsorbed in the matrix. The partition function for a particular matrix and a particular cavity realization is given by

$$
\begin{aligned}
\Xi_{1}\left(\mathbf{q}^{N_{0}}, \mathbf{Q}^{N_{0^{\prime}}}\right) & =\sum_{N_{1}=0}^{\infty} \frac{z_{1}^{N_{1}}}{N_{1} !} \int \mathrm{d} \mathbf{r}^{N_{1}} e^{-\beta H_{10}} \\
& =\sum_{N_{1}=0}^{\infty} \frac{z_{1}^{N_{1}}}{N_{1} !}\left[\int \mathrm{d} \mathbf{r} \exp \left[-\beta \sum_{j=1}^{N_{0}} u_{10}\left(\left|\mathbf{r}_{1}-\mathbf{q}_{j}\right|\right) \theta\left(\mathbf{q}_{j} ; \mathbf{Q}^{N_{0^{\prime}}}\right)\right]\right]^{N_{1}} \\
& =\exp \left\{z_{1} \int \mathrm{d} \mathbf{r}_{1} \exp \left[-\beta \sum_{j=1}^{N_{0}} u_{10}\left(\left|\mathbf{r}_{1}-\mathbf{q}_{j}\right|\right) \theta\left(\mathbf{q}_{j} ; \mathbf{Q}^{N_{0^{\prime}}}\right)\right]\right\}
\end{aligned}
$$

For this model, we need to carry out the averages over matrix and cavity configurations. We will perform these averages in two steps, first over the matrix configurations and then over cavity configurations. The averaging over matrix realizations leads to,

$$
\begin{aligned}
\Omega_{1}\left(\mathbf{Q}^{N_{0^{\prime}}}\right) & =-k T\left\langle\ln \Xi_{1}\left(\mathbf{q}^{N_{0}}, \mathbf{Q}^{N_{0^{\prime}}}\right)\right\rangle_{0} \\
& =\frac{-k T z_{1}}{\Xi_{0}} \sum_{N_{0}=0}^{\infty} \frac{z_{0}^{N_{0}}}{N_{0} !} \int \mathrm{d} \mathbf{q}^{N_{0}} \int \mathrm{d} \mathbf{r}_{1} \exp \left[-\beta \sum_{j=1}^{N_{0}} u_{10}\left(\left|\mathbf{r}_{1}-\mathbf{q}_{j}\right|\right) \theta\left(\mathbf{q}_{j} ; \mathbf{Q}^{N_{0^{\prime}}}\right)\right] \\
& =\frac{-k T z_{1}}{\Xi_{0}} \int \mathrm{d} \mathbf{r}_{1} \exp \left\{z_{0} \int \mathrm{d} \mathbf{q}_{1} \exp \left[-\beta u_{10}\left(\left|\mathbf{r}_{1}-\mathbf{q}_{1}\right|\right) \theta\left(\mathbf{q}_{1} ; \mathbf{Q}^{N_{0^{\prime}}}\right)\right]\right\}
\end{aligned}
$$

The average over cavity realizations is more involved. With the help of the property of the Heaviside function given in equation (84), we first rewrite the integrand on the RHS of the third equality of equation (114) as,

$$
\exp \left[-\beta u_{10}\left(\left|\mathbf{r}_{\mathbf{1}}-\mathbf{q}_{1}\right|\right) \theta\left(\mathbf{q}_{1} ; \mathbf{Q}^{N_{0^{\prime}}}\right)\right]=1-\theta\left(\mathbf{q}_{1} ; \mathbf{Q}^{N_{0^{\prime}}}\right)+\theta\left(\mathbf{q}_{1} ; \mathbf{Q}^{N_{0^{\prime}}}\right) \exp \left[-\beta u_{10}\left(\left|\mathbf{r}_{\mathbf{1}}-\mathbf{q}_{1}\right|\right)\right]
$$


Using equation (115), we can rewrite the integral over $\mathbf{q}_{1}$ in equation (114) as

$$
\begin{aligned}
\int \mathrm{d} \mathbf{q}_{1} \exp [-\beta & \left.u_{10}\left(\left|\mathbf{r}_{1}-\mathbf{q}_{1}\right|\right) \theta\left(\mathbf{q}_{1} ; \mathbf{Q}^{N_{0^{\prime}}}\right)\right]=V-\int_{V} \mathrm{~d} \mathbf{q}_{1} \theta\left(\mathbf{q}_{1} ; \mathbf{Q}^{N_{0^{\prime}}}\right) \\
& =\int_{V} \mathrm{~d} \mathbf{q}_{1} \theta\left(\mathbf{q}_{1} ; \mathbf{Q}^{N_{0^{\prime}}}\right) \exp \left[-\beta u_{10}\left(\left|\mathbf{r}_{1}-\mathbf{q}_{1}\right|\right)\right] \\
& =V-\int_{V} \mathrm{~d} \mathbf{q}_{1} \theta\left(\mathbf{q}_{1} ; \mathbf{Q}^{N_{0^{\prime}}}\right)+\left(\int_{V} \mathrm{~d} \mathbf{q}_{1}-\int_{V_{\sigma_{0}\left(\mathbf{r}_{1}\right)}} \mathrm{d} \mathbf{q}_{1}\right) \theta\left(\mathbf{q}_{1} ; \mathbf{Q}^{N_{0^{\prime}}}\right) \\
& =V-\int_{V_{\sigma_{0}\left(\mathbf{r}_{1}\right)}} \mathrm{d} \mathbf{q}_{1} \theta\left(\mathbf{q}_{1} ; \mathbf{Q}^{N_{0^{\prime}}}\right) .
\end{aligned}
$$

With the help of equation (116), equation (114) is simplified to

$$
\Omega_{1}\left(\mathbf{Q}^{N_{0^{\prime}}}\right)=-k T z_{1} \int \mathrm{d} \mathbf{r}_{1} \exp \left[-z_{0} \int_{V_{\sigma_{0}\left(\mathbf{r}_{1}\right)}} \mathrm{d} \mathbf{q}_{1} \theta\left(\mathbf{q}_{1} ; \mathbf{Q}^{N_{0^{\prime}}}\right)\right]
$$

Now, we proceed to perform the average over the cavity realizations,

$$
\Omega=\left\langle\Omega_{1}\left(\mathbf{Q}^{N_{0^{\prime}}}\right)\right\rangle_{0^{\prime}}=-k T z_{1} \int \mathrm{d} \mathbf{r}_{1}\left\langle\exp \left[-z_{0} \int_{V_{\sigma_{0}\left(\mathbf{r}_{1}\right)}} \mathrm{d} \mathbf{q}_{1} \theta\left(\mathbf{q}_{1} ; \mathbf{Q}^{N_{0^{\prime}}}\right)\right]\right\rangle_{0^{\prime}}
$$

To continue the calculation, we expand the exponential on the RHS of equation (118),

$$
\left\langle\exp \left[-z_{0} \int_{V_{\sigma_{0}\left(\mathbf{r}_{1}\right)}} \mathrm{d} \mathbf{q}_{1} \theta\left(\mathbf{q}_{1} ; \mathbf{Q}^{N_{0^{\prime}}}\right)\right]\right\rangle=\frac{1}{\Xi_{0^{\prime}}} \sum_{N_{0^{\prime}}}^{\infty} \frac{z_{0^{\prime}}^{N_{0^{\prime}}}}{N_{0^{\prime}} !} \int \mathrm{d} \mathbf{Q}^{N_{0^{\prime}}} \sum_{M=0}^{\left(-z_{0}\right)^{M}}\left[\int \mathrm{d} \mathbf{q}_{1} \theta\left(\mathbf{q}_{1} ; \mathbf{Q}^{N_{0^{\prime}}}\right)\right]_{V_{\sigma_{0}\left(\mathbf{r}_{1}\right)}}^{M} .
$$

It should be pointed out that the analytical result cannot be obtained for the above expression in the general case of an arbitrary value of $\sigma_{0}$. Nevertheless, here we show that a close result can be obtained when $\sigma_{0}$ is infinitesimal, i.e., $\sigma_{0} \rightarrow \delta_{0}$. In this case, we have

$$
\begin{aligned}
& {\left[\int_{V_{\sigma_{0}\left(\mathbf{r}_{1}\right)}} \mathrm{d} \mathbf{q}_{1} \theta\left(\mathbf{q}_{1} ; \mathbf{Q}^{N_{0^{\prime}}}\right)\right]^{M}=} \\
& \quad=\int_{V_{\sigma_{0}\left(\mathbf{r}_{1}\right)}} \mathrm{d} \mathbf{q}_{1} \int_{V_{\sigma_{0}\left(\mathbf{r}_{1}\right)}} \mathrm{d} \mathbf{q}_{2} \ldots \int_{V_{\sigma_{0}\left(\mathbf{r}_{1}\right)}} \mathrm{d} \mathbf{q}_{M}\left[\theta\left(\mathbf{q}_{1} ; \mathbf{Q}^{N_{0^{\prime}}}\right) \theta\left(\mathbf{q}_{2} ; \mathbf{Q}^{N_{0^{\prime}}}\right) \ldots \theta\left(\mathbf{q}_{M} ; \mathbf{Q}^{N_{0^{\prime}}}\right)\right] \\
& =\left[V_{\sigma_{0}}\left(\mathbf{r}_{1}\right) \theta\left(\mathbf{r}_{1} ; \mathbf{Q}^{N_{0^{\prime}}}\right)\right]^{M}=\theta\left(\mathbf{r}_{1} ; \mathbf{Q}^{N_{0^{\prime}}}\right)\left(\frac{\pi \sigma_{0}^{3}}{6}\right)^{M}, \quad(M=1,2,3, \ldots) .
\end{aligned}
$$


Substituting equation (120) into equation (119), we finally obtain,

$$
\begin{aligned}
\left\langle\exp \left[-z_{0} \int_{V_{\sigma_{0}}\left(\mathbf{r}_{1}\right)} \mathrm{d} \mathbf{q}_{1} \theta\left(\mathbf{q}_{1} ; \mathbf{Q}^{N_{0^{\prime}}}\right)\right]\right\rangle= \\
\quad=1+\sum_{M=1}^{\infty} \frac{\left(-z_{0}\right)^{M}}{M !}\left(\frac{p i \sigma_{0}^{3}}{6}\right)^{M} \frac{1}{\Xi_{0^{\prime}}} \sum_{N_{0^{\prime}}=0}^{\infty} \frac{z_{0^{\prime}}^{N_{0^{\prime}}}}{N_{0^{\prime}} !} \int \mathrm{d} \mathbf{Q}^{N_{0^{\prime}}} \theta\left(\mathbf{q}_{1} ; \mathbf{Q}^{N_{0^{\prime}}}\right) \\
=1+\left(\mathrm{e}^{-\pi \sigma_{0}^{3} z_{0} / 6}-1\right) \frac{1}{\Xi_{0^{\prime}}} \sum_{N_{0^{\prime}}=0}^{\infty} \frac{z_{0^{\prime}}^{N_{0^{\prime}}}}{N_{0^{\prime}} !}\left(V-\frac{\pi \sigma_{0^{\prime}}^{3}}{6}\right)^{N_{0^{\prime}}}=1+\left(\mathrm{e}^{-\pi \sigma_{0}^{3} z_{0} / 6}-1\right) \mathrm{e}^{-\pi \sigma_{0}^{3} z_{0} / 6}
\end{aligned}
$$

Substituting equation (121) into equation (118), we finally obtain,

$$
\begin{aligned}
\Omega & =-k T z_{1} V\left[1+\left(\mathrm{e}^{-\pi \sigma_{0}^{3} z_{0} / 6}-1\right) \mathrm{e}^{-\pi \sigma_{0}^{3} z_{0} / 6}\right] \\
& =-k T z_{1} V\left[1+\left(\mathrm{e}^{-\pi \sigma_{0}^{3} \rho_{0} / 6}-1\right) \mathrm{e}^{-\pi \sigma_{0}^{3} \rho_{0} / 6}\right],
\end{aligned}
$$

and

$$
P=-\frac{\Omega}{V}=k T z_{1}\left[1+\left(\mathrm{e}^{-\pi \delta_{0}^{3} \rho_{0} / 6}-1\right) \mathrm{e}^{-\pi \sigma_{0^{\prime}}^{3} \rho_{0^{\prime}} / 6}\right] .
$$

As one can anticipate intuitively, equation (123) reduces to the result of the random hard sponge model [see equation (67)] in the limit of $\delta_{0}^{3} \rho_{0} \rightarrow \infty$. The density and the diameter of the cavity in the templated matrix model are $\rho_{0^{\prime}}$ and $\sigma_{0^{\prime}}$ respectively.

The fluid one-body distribution function for a particular matrix realization and a particular cavity realization is given by,

$$
\rho_{1}^{(1)}\left(\mathrm{r}_{1}, \mathrm{q}^{N_{0}}, \mathrm{Q}^{N_{0^{\prime}}}\right)=z_{1} \exp \left[-\beta \sum_{j=1}^{N_{0}} u_{10}\left(\left|\mathrm{r}_{1}-\mathrm{q}_{j}\right|\right) \theta\left(\mathrm{q}_{j} ; \mathrm{Q}^{N_{0^{\prime}}}\right)\right] .
$$

Again, first we carry out the average over matrix realization,

$$
\begin{aligned}
\rho_{1}^{(1)}\left(\mathrm{r}_{1}, \mathrm{Q}^{N_{0^{\prime}}}\right) & =\left\langle\rho_{1}^{(1)}\left(\mathrm{r}_{1}, \mathrm{q}^{N_{0}}, \mathrm{Q}^{N_{0^{\prime}}}\right)\right\rangle_{0} \\
& =\frac{z_{1}}{\Xi_{0}} \sum_{N_{0}=0}^{\infty} \frac{z_{0}^{N_{0}}}{N_{0} !} \int \mathrm{dq}{ }^{N_{0}} \exp \left[-\beta \sum_{j=1}^{N_{0}} u_{10}\left(\left|\mathrm{r}_{1}-\mathrm{q}_{j}\right|\right) \theta\left(\mathrm{q}_{j} ; \mathrm{Q}^{N_{0^{\prime}}}\right)\right] \\
& =\frac{z_{1}}{\Xi_{0}} \sum_{N_{0}=0}^{\infty} \frac{z_{0}^{N_{0}}}{N_{0} !}\left\{\int \mathrm{dq} \exp \left[-\beta u_{10}\left(\left|\mathrm{r}_{1}-\mathrm{q}_{1}\right|\right) \theta\left(\mathrm{q}_{1} ; \mathrm{Q}^{N_{0^{\prime}}}\right)\right]\right\}^{N_{0}} \\
& =\frac{z_{1}}{\Xi_{0}} \exp \left\{z_{0} \int \mathrm{dq}_{1} \exp \left[-\beta u_{10}\left(\left|\mathrm{r}_{1}-\mathrm{q}_{1}\right|\right) \theta\left(\mathrm{q}_{1} ; \mathrm{Q}^{N_{0^{\prime}}}\right)\right]\right\} \\
& =z_{1} \exp \left[z_{0} \int \mathrm{dq}_{1} \theta\left(\mathrm{q}_{1} ; \mathrm{Q}^{N_{0^{\prime}}}\right)\right] .
\end{aligned}
$$

To get the result in equation (125), equation (116) is used again. Now, averaging over the cavity realizations, we obtain

$$
\rho_{1}=\left\langle\left\{\rho_{1}^{(1)}\left(\mathbf{r}_{1}, \mathbf{q}^{N_{0}}\right\rangle_{0^{\prime}}=z_{1}\left\langle\exp \left[-z_{0} \int_{V_{\sigma_{0}}\left(\mathbf{r}_{1}\right)} \operatorname{d} \mathbf{q}_{1} \theta\left(\mathbf{q}_{1} ; \mathbf{Q}^{N_{0^{\prime}}}\right)\right]\right\rangle_{0^{\prime}} .\right.\right.
$$


Again we encounter the same quantity as that in equation (119). Only in the limit of the diameter of matrix particle being infinitesimal, $\sigma_{0} \rightarrow \delta_{0}$, can we obtain the following closed analytical result,

$$
\rho_{1}=z_{1}\left[1+\left(\mathrm{e}^{-\pi \sigma_{0}^{3} \rho_{0} / 6}-1\right) \mathrm{e}^{-\pi \sigma_{0}^{3} \rho_{0} / 6}\right]
$$

The Helmholtz free energy is given by

$$
\frac{F}{V}=k T \rho_{1}\left[\ln \frac{\rho_{1} \Lambda_{1}^{3}}{1+\left(\mathrm{e}^{-\pi \delta_{0}^{3} \rho_{0} / 6}-1\right) \mathrm{e}^{-\pi \sigma_{0^{\prime}}^{3} \rho_{0^{\prime}} / 6}}-1\right] .
$$

We proceed now to calculate the two-body fluid-matrix distribution function. For a particular matrix realization and for a particular cavity realization, we have

$$
\rho_{10}^{(2)}\left(\mathbf{r}, \mathbf{q} ; \mathbf{q}^{N_{0}}, \mathbf{Q}^{N_{0^{\prime}}}\right)=\rho_{1}^{(1)}\left(\mathbf{r} ; \mathbf{q}^{N_{0}}, \mathrm{Q}^{N_{0^{\prime}}}\right) \sum_{j=1}^{N_{0}} \delta\left(\mathbf{q}_{j}-\mathbf{q}\right) \theta\left(\mathbf{q}_{j} ; \mathbf{Q}^{N_{0^{\prime}}}\right) .
$$

The averaging over the matrix realizations leads to

$$
\begin{aligned}
\rho_{10}^{(2)}\left(\mathbf{r}, \mathbf{q} ; \mathbf{Q}^{N_{0^{\prime}}}\right)= & \left\langle\rho_{10}^{(2)}\left(\mathbf{r}, \mathbf{q} ; \mathbf{q}^{N_{0}}, \mathbf{Q}^{N_{0^{\prime}}}\right)\right\rangle_{0} \\
= & \frac{1}{\Xi_{0}} \sum_{N_{0}=0}^{\infty} \frac{z_{0}^{N_{0}}}{\left(N_{0}-1\right) !} \int \mathrm{d} \mathbf{q}^{N_{0}} \delta\left(\mathbf{q}_{1}-\mathbf{q}\right) \theta\left(\mathbf{q}_{1} ; \mathbf{Q}^{N_{0^{\prime}}}\right) \rho_{1}^{(1)}\left(\mathbf{r} ; \mathbf{q}^{N_{0}}, \mathbf{Q}^{N_{0^{\prime}}}\right) \\
= & \frac{z_{1} \theta\left(\mathbf{q} ; \mathbf{Q}^{N_{0}}\right) \exp \left[-\beta u_{10}(|\mathbf{r}-\mathbf{q}|) \theta\left(\mathbf{q} ; \mathbf{Q}^{N_{0^{\prime}}}\right)\right]}{\Xi_{0}} \\
& \left.\times \sum_{N_{0}=0}^{\infty} \frac{z_{0}^{N_{0}}}{\left(N_{0}-1\right) !} \iint \mathrm{d} \mathbf{q}_{2} \exp \left[-\beta u_{10}\left(\left|\mathbf{r}-\mathbf{q}_{2}\right|\right) \theta\left(\mathbf{q}_{2} ; \mathbf{Q}^{N_{0^{\prime}}}\right)\right]\right\}^{N_{0}-1} \\
= & \frac{z_{1} z_{0} \theta\left(\mathbf{q} ; \mathbf{Q}^{N_{0^{\prime}}}\right) \exp \left[-\beta u_{10}(|\mathbf{r}-\mathbf{q}|) \theta\left(\mathbf{q} ; \mathbf{Q}^{N_{0^{\prime}}}\right)\right]}{\Xi_{0}} \\
& \times \exp \left\{z_{0} \int \mathrm{d} \mathbf{q} 2 \exp \left[-\beta u_{10}\left(\left|\mathbf{r}-\mathbf{q}_{2}\right|\right) \theta\left(\mathbf{q}_{2} ; \mathbf{Q}^{N_{0^{\prime}}}\right)\right]\right\}^{N_{0}-1} \\
= & z_{1} z_{0} \theta\left(\mathbf{q} ; \mathbf{Q}^{N_{0^{\prime}}}\right) \exp \left[-\beta u_{10}(|\mathbf{r}-\mathbf{q}|) \theta\left(\mathbf{q} ; \mathbf{Q}^{N_{0^{\prime}}}\right)\right] \exp \left[-z_{0} \int \mathrm{d} \mathbf{q}_{2} \theta\left(\mathbf{q}_{2} ; \mathbf{Q}^{N_{0^{\prime}}}\right)\right] \\
= & z_{1} z_{0} \theta\left(\mathbf{q} ; \mathbf{Q}^{N_{0^{\prime}}}\right) \exp \left[-\beta u_{10}(|\mathbf{r}-\mathbf{q}|)\right] \exp \left[-z_{0} \int \mathrm{d} \mathbf{q}_{2} \theta\left(\mathbf{q}_{2} ; \mathbf{Q}^{N_{0^{\prime}}}\right)\right]
\end{aligned}
$$

In going from the fourth equality to the fifth one in equation (130), equation (116) was used and from the fifth to the sixth, equation (84) was used. The averaging over the cavity realizations now leads to

$$
\begin{aligned}
& \rho_{10}^{(2)}(\mathbf{r}, \mathbf{q})=\left\langle\rho_{10}^{(2)}\left(\mathbf{r}, \mathbf{q} ; \mathbf{Q}^{N_{0^{\prime}}}\right)\right\rangle_{0}=z_{1} z_{0} \mathrm{e}^{-\beta u_{10}(|\mathbf{r}-\mathbf{q}|)}\left\langle\theta\left(\mathbf{q} ; \mathbf{Q}^{N_{0^{\prime}}}\right) \exp \left[-z_{0} \underset{V_{\sigma_{0}(\mathrm{r})}}{\int} \mathrm{d} \mathbf{q}_{2} \theta\left(\mathbf{q}_{2} ; \mathbf{Q}^{N_{0^{\prime}}}\right)\right]\right\rangle_{0^{\prime}} \\
& =z_{1} z_{0} \mathrm{e}^{-\beta u_{10}(|\mathbf{r}-\mathbf{q}|)}\left\langle\theta\left(\mathbf{q} ; \mathbf{Q}^{N_{0^{\prime}}}\right) \sum_{M=0}^{\infty} \frac{1}{M !}\left[-z_{0} \int_{V_{\sigma_{0}(\mathrm{r})}} \mathrm{d} \mathbf{q}_{2} \theta\left(\mathbf{q}_{2} ; \mathbf{Q}^{N_{0^{\prime}}}\right)\right]^{M}\right\rangle_{0^{\prime}} \\
& =z_{1} z_{0} \mathrm{e}^{-\beta u_{10}(|\mathbf{r}-\mathbf{q}|)}\left\{\left\langle\theta\left(\mathbf{q} ; \mathbf{Q}^{N_{0^{\prime}}}\right)\right\rangle_{0}+\left\langle\theta\left(\mathbf{q} ; \mathbf{Q}^{N_{0^{\prime}}}\right) \sum_{M=0}^{\infty} \frac{1}{M !}\left[-z_{0} \iint_{V_{\sigma_{0}(\mathrm{r})}} \mathrm{d} \mathbf{q}_{2} \theta\left(\mathbf{q}_{2} ; \mathbf{Q}^{N_{0^{\prime}}}\right)\right]^{M}\right\rangle\right\}
\end{aligned}
$$




$$
\begin{aligned}
& =z_{1} z_{0} \mathrm{e}^{-\beta u_{10}(|\mathbf{r}-\mathbf{q}|)}\left[\left\langle\theta\left(\mathbf{q} ; \mathbf{Q}^{N_{0^{\prime}}}\right)\right\rangle_{0}+\left\langle\theta\left(\mathbf{q} ; \mathbf{Q}^{N_{0^{\prime}}}\right) \theta\left(\mathbf{r} ; \mathbf{Q}^{N_{0^{\prime}}}\right) \sum_{M=1}^{\infty} \frac{1}{M !}\left(\frac{-\pi \delta_{0}^{3} z_{0}}{6}\right)^{M}\right\rangle_{0}\right] \\
& =z_{1} z_{0} \mathrm{e}^{-\beta u_{10}(|\mathbf{r}-\mathbf{q}|)}\left[\left\langle\theta\left(\mathbf{q} ; \mathbf{Q}^{N_{0^{\prime}}}\right)\right\rangle_{0}+\left(\mathrm{e}^{-\pi \delta_{0}^{3} z_{0} / 6}-1\right)\left\langle\theta\left(\mathbf{q} ; \mathbf{Q}^{N_{0^{\prime}}}\right) \theta\left(\mathbf{r} ; \mathbf{Q}^{N_{0^{\prime}}}\right)\right\rangle_{0}\right] \\
& =z_{1} z_{0} \mathrm{e}^{-\beta u_{10}(|\mathbf{r}-\mathbf{q}|)}\left[\mathrm{e}^{-\pi \sigma_{0^{\prime}}^{3} z_{0^{\prime}} / 6}+\left(\mathrm{e}^{-\pi \delta_{0}^{3} z_{0} / 6}-1\right) \mathrm{e}^{-z_{0^{\prime}} V_{d b}(|\mathbf{r}-\mathbf{q}|)}\right] .
\end{aligned}
$$

Equations (103) and (107) are used to obtain the last equality of equation (131).

Now, we consider the fluid-cavity two-body distribution function which has the following form for a particular matrix and a particular cavity realization,

$$
\rho_{10}^{(2)}\left(\mathbf{r}, \mathbf{Q} ; \mathbf{q}^{N_{0}} \mathbf{Q}^{N_{0^{\prime}}}\right)=\rho_{1}^{(1)}\left(\mathbf{r} ; \mathbf{q}^{N_{0}}, \mathbf{Q}^{N_{0^{\prime}}}\right) \sum_{j=1}^{N_{0^{\prime}}} \delta\left(\mathbf{Q}_{j}-\mathbf{Q}\right) .
$$

Taking the average over matrix realizations, we have

$$
\begin{aligned}
\rho_{10^{\prime}}^{(2)}\left(\mathbf{r}, \mathbf{Q} ; \mathbf{Q}^{N_{0^{\prime}}}\right) & =\left\langle\rho_{1} 0^{(2)}\left(\mathbf{r}, \mathbf{Q} ; \mathbf{q}^{N_{0}}, \mathbf{Q}^{N_{0^{\prime}}}\right)\right\rangle_{0}=\left\langle\rho_{1}^{(1)}\left(\mathbf{r} ; \mathbf{q}^{N_{0}}, \mathbf{Q}^{N_{0^{\prime}}}\right)\right\rangle_{0} \sum_{j=1}^{N_{0^{\prime}}} \delta\left(\mathbf{Q}_{j}-\mathbf{Q}\right) \\
& =z_{1} \exp \left[-z_{0} \int_{V \sigma_{0}(\mathbf{r})} \mathrm{d} \mathbf{q}_{1} \theta\left(\mathbf{q}_{1} ; \mathbf{Q}^{\left.N_{0^{\prime}}\right)}\right] \sum_{j=1}^{N_{0^{\prime}}} \delta\left(\mathbf{Q}_{j}-\mathbf{Q}\right) .\right.
\end{aligned}
$$

The averaging over the cavity realizations yields,

$$
\begin{aligned}
\rho_{10^{\prime}}^{(2)}(\mathbf{r}-\mathbf{Q}) & =\left\langle\rho_{1} 0^{\prime(2)}\left(\mathbf{r}, \mathbf{Q} ; \mathbf{Q}^{N_{0^{\prime}}}\right)\right\rangle_{0^{\prime}} \\
& =\frac{z_{1}}{\Xi_{0^{\prime}}} \sum_{N_{0^{\prime}}}^{\infty} \frac{z_{0^{\prime}}^{N_{0^{\prime}}}}{\left(N_{0^{\prime}}-1\right) !} \int \mathrm{d} \mathbf{Q}^{N_{0^{\prime}}-1} \exp \left[-z_{0} \iint_{V \sigma_{0}(\mathbf{r})} \mathrm{d} \mathbf{q}_{1} \theta\left(\mathbf{q}_{1} ; \mathbf{Q}^{N_{0^{\prime}}}\right)\right] \\
& =\frac{z_{1}}{\Xi_{0^{\prime}}} \sum_{N_{0^{\prime}}}^{\infty} \frac{z_{0^{\prime}}^{N_{0^{\prime}}}}{\left(N_{0^{\prime}}-1\right) !} \int \mathrm{d} \mathbf{Q}^{N_{0^{\prime}}-1} \sum_{M=0}^{\infty} \frac{1}{M !}\left[-z_{0} \int_{V \sigma_{0}(\mathbf{r})} \mathrm{d} \mathbf{q}_{1} \theta\left(\mathbf{q}_{1} ; \mathbf{Q}^{N_{0^{\prime}}}\right)\right]^{M} \\
& =z_{1}\left[z_{0}+\sum_{M=0}^{\infty} \frac{1}{M !}\left(-\frac{\pi \delta_{0}^{3} z_{0}}{6}\right)^{M} \frac{1}{\Xi_{0^{\prime}}} \sum_{N_{0^{\prime}}}^{\infty} \frac{z_{0^{\prime}}^{N_{0^{\prime}}}}{\left(N_{0^{\prime}}-1\right) !} \int \mathrm{d} \mathbf{Q}^{N_{0^{\prime}}-1} \theta\left(\mathbf{r} ; \mathbf{Q}^{N_{0^{\prime}}}\right)\right] \\
& =z_{1}\left[z_{0}+\left(\mathrm{e}^{-\pi \delta_{0}^{3} z_{0} / 6}-1\right) \frac{\mathrm{e}^{-\beta u_{00^{\prime}}(|\mathbf{r}-\mathbf{Q}|)}}{\Xi_{0^{\prime}}} \sum_{N_{0^{\prime}}=0}^{\infty} \frac{z_{0^{\prime}}^{N_{0^{\prime}}}}{\left(N_{0^{\prime}}-1\right) !}\left(V-\frac{\pi \sigma_{0^{\prime}}^{3}}{6}\right)^{N_{0^{\prime}}-1}\right] \\
& =z_{1} z_{0}\left[1+\mathrm{e}^{-\pi \delta_{0}^{3} z_{0} / 6}\left(\mathrm{e}^{-\pi \delta_{0}^{3} z_{0} / 6}-1\right) \mathrm{e}^{-\beta u_{00^{\prime}}(|\mathbf{r}-\mathbf{Q}|)}\right] .
\end{aligned}
$$

In going from the third equality in equation (134) to the fourth one, we take the limit of infinitesimal matrix particle limit, i.e., $\sigma_{0} \rightarrow \delta_{0}$. In the limit of $\delta_{0}^{3} z_{0} \rightarrow \infty$, equation (83) reduces to the result of fluid-matrix two-body distribution function of the hard sponge model (see equation (77)).

The fluid-fluid two-body distribution function for a particular matrix realization and a particular cavity realization is given by

$$
\rho_{11}^{(2)}\left(\mathbf{r}_{1}, \mathbf{r}_{2} ; \mathbf{q}^{N_{0}}\right)=\rho_{1}^{(1)}\left(\mathbf{r}_{1} ; \mathbf{q}^{N_{0}}, \mathbf{Q}^{N_{0^{\prime}}}\right) \rho_{1}^{(1)}\left(\mathbf{r}_{2} ; \mathbf{q}^{N_{0}}, \mathbf{Q}^{N_{0^{\prime}}}\right) .
$$


Taking the average over matrix realizations, we have

$$
\begin{aligned}
\rho_{11}^{(2)}\left(\mathbf{r}_{1}, \mathbf{r}_{2} ; \mathbf{Q}^{N_{0^{\prime}}}\right) & =\left\langle\rho_{11}^{(2)}\left(\mathbf{r}_{1}, \mathbf{r}_{2} ; \mathbf{q}^{N_{0}}, \mathbf{Q}^{N_{0^{\prime}}}\right)\right\rangle_{0} \\
& =\frac{z_{1}^{2}}{\Xi_{0}} \sum_{N_{0}=0}^{\infty} \frac{z_{0}^{N_{0}}}{N_{0} !} \int \mathrm{d} \mathbf{q}^{N_{0}} \exp \left[-\beta \sum_{j=1}^{N_{0}} \theta\left(\mathbf{q}_{j} ; \mathbf{Q}^{N_{0}}\right) \sum_{i=1}^{2} u_{10}\left(\left|\mathbf{r}_{i}-\mathbf{q}_{j}\right|\right)\right] \\
& =\frac{z_{1}^{2}}{\Xi_{0}} \sum_{N_{0}=0}^{\infty} \frac{z_{0}^{N_{0}}}{N_{0} !}\left\{\int \mathrm{d} \mathbf{q}_{1} \exp \left[-\beta \theta\left(\mathbf{q}_{1} ; \mathbf{Q}^{N_{0^{\prime}}}\right) \sum_{i=1}^{2} u_{10}\left(\left|\mathbf{r}_{i}-\mathbf{q}_{1}\right|\right)\right]\right\}^{N_{0}} \\
& =\frac{z_{1}^{2}}{\Xi_{0}} \exp \left\{z_{0} \int \mathrm{d} \mathbf{q}_{1} \exp \left[-\beta \theta\left(\mathbf{q}_{1} ; \mathbf{Q}^{N_{0^{\prime}}}\right) \sum_{i=1}^{2} u_{10}\left(\left|\mathbf{r}_{i}-\mathbf{q}_{1}\right|\right)\right]\right\} .
\end{aligned}
$$

Using a technique similar to the one used in deriving equation (116), we straightforwardly obtain,

$$
\int \mathrm{d} \mathbf{q}_{1} \exp \left[-\beta \theta\left(\mathbf{q}_{1} ; \mathbf{Q}^{N_{0}^{\prime}}\right) \sum_{i=1}^{2} u_{10}\left(\left|\mathbf{r}_{i}-\mathbf{q}_{1}\right|\right)\right]=V-\int_{V_{d b}\left(\left|\mathbf{r}_{1}-\mathbf{r}_{2}\right|\right)} \int \mathrm{d} \mathbf{q}_{1} \theta\left(\mathbf{q}_{1} ; \mathbf{Q}^{N_{0^{\prime}}}\right),
$$

where $V_{d b}\left(\left|\mathbf{r}_{1}-\mathbf{r}_{2}\right|\right)$ is the volume of a dumbbell formed by two spheres with a diameter of $\sigma_{0}$ located at $\mathbf{r}_{1}$ and $\mathbf{r}_{2}$. Substituting equation (137) into equation (136), we obtain

$$
\rho_{11}^{2}\left(\mathbf{r}_{1} \mathbf{r}_{2} ; \mathbf{Q}^{N_{0^{\prime}}}\right)=z_{1}^{2} \exp \left[-z_{0} \int_{V_{d b}\left(\left|\mathbf{r}_{1}-\mathbf{r}_{2}\right|\right)} \mathrm{d} \mathbf{q}_{1} \theta\left(\mathbf{q}_{1} ; \mathbf{Q}^{N_{0^{\prime}}}\right)\right]
$$

Finally, we take the average over the cavity realizations,

$$
\rho_{11}^{(2)}\left(\left|\mathbf{r}_{1}-\mathbf{r}_{2}\right|\right)=\left\langle\rho_{11}^{(2)}\left(\mathbf{r}_{1}, \mathbf{r}_{2} ; \mathbf{Q}^{N_{0^{\prime}}}\right)\right\rangle_{0^{\prime}}=z_{1}^{2}\left\langle\exp \left[-z_{0} \int_{V_{d b}\left(\left|\mathbf{r}_{1}-\mathbf{r}_{2}\right|\right)} \operatorname{d} \mathbf{q}_{1} \theta\left(\mathbf{q}_{1} ; \mathbf{Q}^{\left.N_{0^{\prime}}\right)}\right]\right\rangle_{0^{\prime}}(13\right.
$$

We were not able to analytically calculate the average in equation (139) for an arbitrary value of $\left|\mathbf{r}_{1}-\mathbf{r}_{2}\right|$. In the limit of infinitesimal matrix particles and for $\left|\mathbf{r}_{1}-\mathbf{r}_{2}\right|=0$, equation (139) becomes

$$
\begin{aligned}
\rho_{11}^{(2)}\left(\left|\mathbf{r}_{1}-\mathbf{r}_{2}\right|=0\right) & =z_{1}^{2} \lim _{\sigma_{0} \rightarrow \delta_{0}}\left\langle\exp \left[-z_{0} \int_{V \sigma_{0}(\mathbf{r})} \mathrm{d} \mathbf{q}_{1} \theta\left(\mathbf{q}_{1} ; \mathbf{Q}^{N_{0^{\prime}}}\right)\right]\right\rangle_{0^{\prime}} \\
& =1+\left(\mathrm{e}^{-\pi \delta_{0}^{3} z_{0} / 6}-1\right) \mathrm{e}^{-\pi \delta_{0}^{3} z_{0} / 6},
\end{aligned}
$$

where equation (121) was used in order to obtain the final result. Again, the result in equation (140) reduces to that of the hard sponge model in the limit of $\delta_{0}^{3} z_{0} \rightarrow \infty$ (see equation (76)).

\section{B. Van Tassel model}

The construction of the templated matrix proposed by Van Tassel is illustrated in figure 6 . Since the matrix of Van Tassel model is generated from the configurations of an equilibrium fluid mixture, the templated matrix thus generated is characterized by the matrix-matrix, $H_{00}$, templatetemplate, $H_{0^{\prime} 0^{\prime}}$, and matrix-template, $H_{00^{\prime}}$, interactions. 


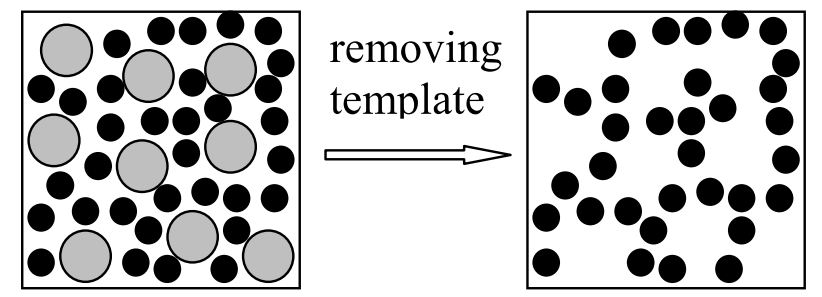

Figure 6. Sketch of Van Tassel's model of templated matrices: matrix particles represented by black spheres and template particles represented by grey spheres.

First, we consider the model generated from a binary HS mixture. In this case,

$$
\begin{aligned}
H_{00} & =\frac{1}{2} \sum_{i=1}^{N_{0}} \sum_{j=1, j \neq i}^{N_{0}} u_{00}\left(\left|\mathbf{q}_{i}-\mathbf{q}_{j}\right|\right), \\
u_{00}\left(\left|\mathbf{q}_{i}-\mathbf{q}_{j}\right|\right) & =\left\{\begin{array}{cc}
\infty, & \left|\mathbf{q}_{i}-\mathbf{q}_{j}\right|<\sigma_{0}, \\
0, & \left|\mathbf{q}_{i}-\mathbf{q}_{j}\right| \geqslant \sigma_{0},
\end{array}\right. \\
H_{0^{\prime} 0^{\prime}} & =\frac{1}{2} \sum_{i=1}^{N_{0^{\prime}}} \sum_{j=1, j \neq i}^{N_{0^{\prime}}} u_{0^{\prime} 0^{\prime}}\left(\left|\mathbf{Q}_{i}-\mathbf{Q}_{j}\right|\right), \\
u_{0^{\prime} 0^{\prime}}\left(\left|\mathbf{Q}_{i}-\mathbf{Q}_{j}\right|\right) & =\left\{\begin{array}{cc}
\infty, & \left|\mathbf{Q}_{i}-\mathbf{Q}_{j}\right|<\sigma_{0^{\prime}}, \\
0, & \left|\mathbf{Q}_{i}-\mathbf{Q}_{j}\right| \geqslant \sigma_{0^{\prime}},
\end{array}\right. \\
H_{00^{\prime}} & =\sum_{i=1}^{N_{0}} \sum_{j=1, j \neq i}^{N_{0^{\prime}}} u_{00^{\prime}}\left(\left|\mathbf{q}_{i}-\mathbf{Q}_{j}\right|\right), \\
u_{00^{\prime}}\left(\left|\mathbf{q}_{i}-\mathbf{Q}_{j}\right|\right) & =\left\{\begin{array}{cc}
\infty, & \left|\mathbf{q}_{i}-\mathbf{Q}_{j}\right|<\sigma_{00^{\prime}}, \\
0, & \left|\mathbf{q}_{i}-\mathbf{Q}_{j}\right| \geqslant \sigma_{00^{\prime}} .
\end{array}\right.
\end{aligned}
$$

The fluid-matrix interaction is again described by equations (22) and (23) and we set the fluid-fluid interaction to be zero since we always consider an ideal gas. Now, we see that the thermodynamic properties of this model can be easily obtained but no closed analytical results can be easily obtained for different two-body distribution functions. The partition function for a particular templated matrix realization has exactly the same form as that for an IG in a Madden-Gland HS matrix, i.e., equation (49). Using equation (49), we find the following expression for the grand potential averaged over matrix realizations,

$$
\begin{aligned}
\Omega & =\frac{-k T z_{1}}{\Xi_{00^{\prime}}} \sum_{N_{0}=0}^{\infty} \sum_{N_{0^{\prime}}=0}^{\infty} \frac{z_{0}^{N_{0}} z_{0^{\prime}}^{N_{0^{\prime}}}}{N_{0} ! N_{0^{\prime}} !} \int \mathrm{d} \mathbf{q}^{N_{0}} \int \mathrm{d} \mathbf{Q}^{N_{0^{\prime}}} \mathrm{e}^{-\beta\left(H_{00}+H_{0^{\prime} 0^{\prime}}+H_{00^{\prime}}\right)}\left(V-\frac{N_{0} \pi \sigma_{0}^{3}}{6}\right) \\
& =-k T z_{1}\left(V-\frac{\pi \sigma_{0}^{3}}{6} \int \mathrm{d} \mathbf{q}_{1} \rho_{0}\right)=-k T z_{1} V\left(1-\frac{\pi \sigma_{0}^{3} \rho_{0}}{6}\right),
\end{aligned}
$$

where

$$
\Xi_{00^{\prime}}=\sum_{N_{0}=0}^{\infty} \sum_{N_{0^{\prime}}=0}^{\infty} \frac{z_{0}^{N_{0}} z_{0^{\prime}}^{N_{0^{\prime}}}}{N_{0} ! N_{0^{\prime}} !} \int \mathrm{d} \mathbf{q}^{N_{0}} \int \mathrm{d} \mathbf{Q}^{N_{0^{\prime}}} \mathrm{e}^{-\beta\left(H_{00}+H_{0^{\prime} 0^{\prime}}+H_{00^{\prime}}\right)}
$$

is the partition function of the templated matrix and

$$
\rho_{0}=\frac{1}{\Xi_{00^{\prime}}} \sum_{N_{0}=0}^{\infty} \sum_{N_{0^{\prime}}=0}^{\infty} \frac{z_{0}^{N_{0}} z_{0^{\prime}}^{N_{0^{\prime}}}}{\left(N_{0}-1\right) ! N_{0^{\prime}} !} \int \mathrm{d} \mathbf{q}^{N_{0}-1} \int \mathrm{d} \mathbf{Q}^{N_{0^{\prime}}} \mathrm{e}^{-\beta\left(H_{00}+H_{0^{\prime} 0^{\prime}}+H_{00^{\prime}}\right)}
$$


is the density of matrix particles. The pressure is given by

$$
P=\frac{-\Omega}{V}=k T z_{1}\left(1-\frac{\pi \sigma_{0}^{3} \rho_{0}}{6}\right)
$$

Although equation (150) looks formally identical to equation (51), the matrix density in these two equations have different meanings [compare equations (52) and (149)]. Nevertheless, for the same value of matrix density and the same matrix particle size, the thermodynamics of an IG is the same no matter whether it is adsorbed in a HS Madden-Glandt matrix or in a Van Tassel templated matrix generated from a binary HS mixture. We can also put this in another way. For its thermodynamics, an IG does not see the structure difference between a HS Madden-Glandt matrix and a Van Tassel templated matrix. The physical reason for this is that the thermodynamics of an IG is uniquely determined by the volume of the pore space rather than by its shape. With the same values of $\rho_{0}$ and $\sigma_{0}$, the shape pore space in the templated matrix is different from that of HS Madden-Glandt matrix but the two matrices have the same pore volume.

The one-body fluid distribution function for a particular matrix realization is given by

$$
\rho_{1}^{(1)}\left(\mathbf{r} ; \mathbf{q}^{N_{0}}\right)=\left\langle\sum_{i=1}^{N_{1}} \delta\left(\mathbf{r}_{1}-\mathbf{r}\right)\right\rangle_{1}=z_{1} \exp \left[-\beta \sum_{j=1}^{N_{0}} u_{10}\left(\left|\mathbf{r}-\mathbf{q}_{j}\right|\right)\right]
$$

The averaging over matrix realizations yields

$$
\begin{aligned}
& \rho_{1}=\left\langle\rho_{1}^{(1)}\left(\mathbf{r} ; \mathbf{q}^{N_{0}}\right)\right\rangle_{00^{\prime}}=\frac{z_{1}}{\Xi_{00^{\prime}}} \sum_{N_{0}=0}^{\infty} \sum_{N_{0^{\prime}}=0}^{\infty} \frac{z_{0}^{N_{0}} z_{0^{\prime}}^{N_{0^{\prime}}}}{N_{0}^{N_{0^{\prime}}}} N_{0} ! N_{0^{\prime}} ! \\
& \times \int \mathrm{d}^{N_{0}} \int \mathrm{d} \mathbf{Q}^{N_{0^{\prime}}} \mathrm{e}^{-\beta\left(H_{00}+H_{0^{\prime} 0^{\prime}}+H_{00^{\prime}}\right)} \exp \left[-\beta \sum_{j=1}^{N_{0}} u_{10}\left(\left|\mathbf{r}-\mathbf{q}_{j}\right|\right)\right] \\
&= \frac{z_{1}}{\Xi_{00^{\prime}}} \sum_{N_{0}=0}^{\infty} \sum_{N_{0^{\prime}}=0}^{\infty} \frac{z_{0}^{N_{0}} z_{0^{\prime}}^{N_{0^{\prime}}}}{N_{0} ! N_{0^{\prime}} !} \int \mathrm{d} \mathbf{Q}^{N_{0^{\prime}}}\left(\int_{V} \mathrm{~d} \mathbf{q}_{1}-\int_{V_{\sigma_{0}}(\mathbf{r})} \mathrm{d} \mathbf{q}_{1}\right)\left(\int_{V} \mathrm{~d} \mathbf{q}_{2}-\underset{V_{\sigma_{0}}(\mathbf{r})}{\int} \mathrm{d} \mathbf{q}_{2}\right) \ldots \\
& \times\left(\int_{V} \mathrm{~d} \mathbf{q}_{N_{0}}-\iint_{V_{\sigma_{0}}(\mathbf{r})} \mathrm{d} \mathbf{q}_{N_{0}}\right) \\
&\left.\mathrm{V}^{-\beta\left(H_{00}+H_{0^{\prime} 0^{\prime}}+H_{00^{\prime}}\right)}=z_{1} \rho_{0}\right) \\
& z_{1}\left(1-\frac{\pi \sigma_{0}^{3} \rho_{0}}{6}\right) .
\end{aligned}
$$

The result for the adsorbed fluid density as a function of the fluid chemical potential is also identical to that obtained for a HS matrix [see equation (55)]. The Helmholtz free energy is given by

$$
\frac{F}{V}=k T \rho_{1}\left[\ln \frac{\rho_{1} \Lambda_{1}^{3}}{1-\pi \sigma_{0}^{3} \rho_{0} / 6}-1\right]
$$

This result is again identical to that for a HS matrix [compare equation (153) to equation (56)]. All these are the further evidence that the thermodynamic properties of an ideal gas confined in porous media depend only on the accessible pore volume rather than on the pore shape or the structure of the pore space. Therefore, at the same chemical potential, the pressure, the adsorbed density and the free energy of an ideal gas are the same if the matrix packing fraction, i.e., $\eta_{0}=\pi \sigma_{0}^{3} \rho_{0} / 6$, is fixed no matter whether the matrix is a HS one or a templated HS one. 
The fluid-matrix two-body distribution function averaged over matrix realizations is given by

$$
\begin{aligned}
& \rho_{01}^{(2)}(|\mathbf{q}-\mathbf{r}|)=\left\langle\rho_{1}^{(1)}\left(\mathbf{r} ; \mathbf{q}^{N_{0}}\right) \sum_{i=1}^{N_{0}} \delta\left(\mathbf{q}_{i}-\mathbf{q}\right)\right\rangle_{00^{\prime}}=\frac{z_{1} \mathrm{e}^{-\beta u_{10}(|\mathbf{r}-\mathbf{q}|)}}{\Xi_{00^{\prime}}} \sum_{N_{0}=0}^{\infty} \sum_{N_{0^{\prime}}=0}^{\infty} \frac{z_{0}^{N_{0}} z_{0^{\prime}}^{N_{0^{\prime}}}}{\left(N_{0}-1\right) ! N_{0^{\prime}} !} \\
& \times \int \mathrm{d} \mathbf{q}^{N_{0}-1} \int \mathrm{d} \mathbf{Q}^{N_{0^{\prime}}} \mathrm{e}^{-\beta\left(H_{00}+H_{0^{\prime} 0^{\prime}}+H_{00^{\prime}}\right)} \exp \left[-\beta \sum_{j=2}^{N_{0}} u_{10}\left(\left|\mathbf{r}-\mathbf{q}_{j}\right|\right)\right] \\
& =\frac{z_{1} \mathrm{e}^{-\beta u_{10}(|\mathbf{r}-\mathbf{q}|)}}{\Xi_{00^{\prime}}} \sum_{N_{0}=0}^{\infty} \sum_{N_{0^{\prime}}=0}^{\infty} \frac{z_{0}^{N_{0}} z_{0^{\prime}}^{N_{0^{\prime}}}}{\left(N_{0}-1\right) ! N_{0^{\prime}} !} \\
& \times \int \mathrm{d} \mathbf{Q}^{N_{0^{\prime}}}\left(\int_{V} \mathrm{~d} \mathbf{q}_{2}-\underset{V_{\sigma_{0}}(\mathbf{r})}{\int} \mathrm{d} \mathbf{q}_{2}\right)\left(\int_{V} \mathrm{~d} \mathbf{q}_{3}-\underset{V_{\sigma_{0}}(\mathbf{r})}{\int} \mathrm{d} \mathbf{q}_{3}\right) \ldots \\
& \times\left(\int_{V} \mathrm{~d} \mathbf{q}_{N_{0}}-\int_{V_{\sigma_{0}}(\mathbf{r})} \mathrm{d} \mathbf{q}_{N_{0}}\right) \mathrm{e}^{-\beta\left(H_{00}+H_{0^{\prime} 0^{\prime}}+H_{00^{\prime}}\right)} \\
& =z_{1} \mathrm{e}^{-\beta u_{10}(|\mathbf{r}-\mathbf{q}|)}\left(\rho_{0}-\int_{V_{\sigma_{0}}(\mathbf{r})} \mathrm{d} \mathbf{q}_{2} \rho_{00}^{(2)}\left(\left|\mathbf{q}-\mathbf{q}_{2}\right|\right)\right) .
\end{aligned}
$$

In exactly the same way, we obtain the following result for the two-body fluid-template distribution function,

$$
\rho_{0^{\prime} 1}^{(2)}(|\mathbf{Q}-\mathbf{r}|)=\left\langle\rho_{1}^{(1)}\left(\mathbf{r} ; \mathbf{q}^{N_{0}}\right) \sum_{i=1}^{N_{0}} \delta\left(\mathbf{Q}_{i}-\mathbf{Q}\right)\right\rangle_{00^{\prime}}=z_{1}\left(\rho_{0^{\prime}}-\int_{V_{\sigma_{0}}(\mathbf{r})} \mathrm{d} \mathbf{q}_{1} \rho_{0^{\prime} 0}^{(2)}\left(\left|\mathbf{Q}-\mathbf{q}_{1}\right|\right)\right)
$$

The fluid-fluid two-body distribution averaged over matrix realizations is given by

$$
\begin{aligned}
& \rho_{11}^{(2)}\left(\left|\mathbf{r}-\mathbf{r}^{\prime}\right|\right)=\left\langle\rho_{1}^{(1)}\left(\mathbf{r} ; \mathbf{q}^{N_{0}}\right) \rho_{1}^{(1)}\left(\mathbf{r}^{\prime} ; \mathbf{q}^{N_{0}}\right)\right\rangle_{00}=\frac{z_{1}^{2}}{\Xi_{00^{\prime}}} \sum_{N_{0}=0}^{\infty} \sum_{N_{0^{\prime}}=0}^{\infty} \frac{z_{0}^{N_{0}} z_{0^{\prime}}^{N_{0^{\prime}}}}{N_{0} ! N_{0^{\prime}} !} \\
& \times \int \mathrm{d} \mathbf{q}^{N_{0}-1} \int \mathbf{Q}^{N_{0^{\prime}}} \mathrm{e}^{-\beta\left(H_{00}+H_{0^{\prime} 0^{\prime}}+H_{00^{\prime}}\right)} \exp \left\{-\beta \sum_{j=1}^{N_{0}}\left[u_{10}\left(\left|\mathbf{r}-\mathbf{q}_{j}\right|\right)+u_{10}\left(\left|\mathbf{r}^{\prime}-\mathbf{q}_{j}\right|\right)\right]\right\} \\
& =\frac{z_{1}^{2}}{\Xi_{00^{\prime}}} \sum_{N_{0}=0}^{\infty} \sum_{N_{0^{\prime}}=0}^{\infty} \frac{z_{0}^{N_{0}} z_{0^{\prime}}^{N_{0^{\prime}}}}{N_{0} ! N_{0^{\prime}} !} \int \mathrm{d} \mathbf{Q}^{N_{0^{\prime}}}\left(\int_{V} \mathrm{~d} \mathbf{q}_{1}-\int_{V_{d b}\left(\left|\mathbf{r}-\mathbf{r}^{\prime}\right|\right)} \mathrm{d} \mathbf{q}_{1}\right)\left(\int_{V} \mathrm{~d} \mathbf{q}_{2}-\int_{V_{d b}\left(\left|\mathbf{r}-\mathbf{r}^{\prime}\right|\right)} \mathrm{d} \mathbf{q}_{2}\right) \ldots \\
& \times\left(\int_{V} \mathrm{~d} \mathbf{q}_{N_{0}}-\int_{V_{d b}\left(\left|\mathbf{r}-\mathbf{r}^{\prime}\right|\right)} \mathrm{d} \mathbf{q}_{N_{0}}\right) \mathrm{e}^{-\beta\left(H_{00}+H_{0^{\prime} 0^{\prime}}+H_{00^{\prime}}\right)} \\
& =z_{1}^{2}\left(1-\rho_{0} V_{d b}\left(\left|\mathbf{r}-\mathbf{r}^{\prime}\right|\right)+\frac{1}{2} \int_{V_{d b}\left(\left|\mathbf{r}-\mathbf{r}^{\prime}\right|\right)} \mathrm{d} \mathbf{q}_{1} \int_{V_{d b}\left(\left|\mathbf{r}-\mathbf{r}^{\prime}\right|\right)} \mathrm{d}_{\mathbf{q}_{2}} \rho_{00}^{(2)}\left(\left|\mathbf{q}_{1}-\mathbf{q}_{2}\right|\right)\right) .
\end{aligned}
$$

The two-body distribution functions given in equations (154) and (156) are formally identical to those for an IG in a HS Madden-Glandt matrix (see equations (59) and (57)). However, it should be noted that matrix-matrix distribution function, $\rho_{00}^{(2)}\left(\left|\mathbf{q}_{1}-\mathbf{q}_{2}\right|\right)$, in equations (154) and (156) 
is that for a HS mixture while that in equations (59) and (57) is that for an one-component HS system.

Finally, we consider a Van Tassel templated matrix in which

$$
\begin{gathered}
H_{00}=0, \\
H_{0^{\prime} 0^{\prime}}=0,
\end{gathered}
$$

and $H_{00^{\prime}}$ is still described by equations (145) and (146). This matrix is generated by quenching a Widom-Rowlinson mixture [83] and removing then one species (say species $0^{\prime}$ ). This case is the closest to the RHS/RC model discussed in section 5.A. In this case, the partition function for a particular matrix realization has the same expression as that given in equation (24). The grand potential averaged over matrix realizations is given by

$$
\begin{aligned}
\Omega= & -k T\left\langle\ln \Xi_{1}\left(\mathbf{q}^{N_{0}}\right)\right\rangle_{00^{\prime}}=\frac{-k T z_{1}}{\Xi_{00^{\prime}}} \sum_{N_{0}=0}^{\infty} \sum_{N_{0^{\prime}}=0}^{\infty} \frac{z_{0}^{N_{0}} z_{0^{\prime}}^{N_{0^{\prime}}}}{N_{0} ! N_{0^{\prime}} !} \\
& \times \int \mathrm{d} \mathbf{q}^{N_{0}} \int \mathrm{d} \mathbf{Q}^{N_{0}^{\prime}} \mathrm{e}^{-\beta H_{00^{\prime}}} \int \mathrm{d} \mathbf{r}_{1} \exp \left[-\beta \sum_{j=1}^{N_{0}} u_{10}\left(\left|\mathbf{r}_{1}-\mathbf{q}_{j}\right|\right)\right] .
\end{aligned}
$$

I did not succeed in obtaining an analytical result in closed form for the grand potential in this case. It seems that one should also recover the hard-sponge model from this Van Tassel's model (i.e., $H_{00}=0$ and $H_{0^{\prime} 0^{\prime}}=0$ ) in the limits of $\sigma_{0} \rightarrow 0$ and $\rho_{0} \sigma_{0}^{3} \rightarrow \infty$. Since it is not even possible to prove this in the case of an ideal gas, the above conjesture remains an open problem.

\section{Concluding remarks}

In this paper, the adsorption of an ideal gas in a variety of models for random porous media is studied. When a matrix model is not too complicated, it is possible to obtain all the results for thermodynamic functions, one- and two-body distribution functions in closed analytical forms. For more complicated matrix models, even the study of an adsorbed ideal gas becomes quite difficult. For example, analytical results in closed forms have been found for RHS/RC matrix (Zhao-DongLiu model of templated matrices) only in the case of infinitesimal matrix particles. For Van Tassel model of templated matrices, no analytical result has been found in the case that the matrix is built from a Widom-Rowlinson mixture. The integral-equation theory based on Ornstein-Zernike equations for the matrix models considered in the present paper is now available [1,31-33,37]. The application of such a theoretical approach also requires approximate closures. A basic virtue of any good approximation should be its capability of exactly recovering the results of an ideal gas when the fluid-fluid interaction is set to zero. In this work, it is shown that the well-known PercusYevick closure does not possess this virtue when it is applied to the fluids confined in a random hard-sphere matrix. I hope that the compilation of the exact and analytical results for an ideal gas adsorbed in the currently available various matrix models will provide a useful reference.

\section{References}

1. Madden W.G., Glandt E.D., J. Stat. Phys., 1988, 51 , 537.

2. Fanti L.A., Glandt E.D., Madden W.G., J. Chem. Phys. 1990, 93, 5945.

3. Madden W.G., J. Chem. Phys., 1992, 96, 5422.

4. Thompson A. P., Glandt E. D., J. Chem. Phys., 1993, 99, 8325.

5. Ford D.M., Glandt E.D., J. Chem. Phys., 1994, 100, 2391.

6. Ford D.M., Glandt E.D., Phys. Rev. E, 1994, 50, 1280.

7. Ford D.M., Thompson A.P., Glandt E.D., J. Chem. Phys., 1995, 103, 1099.

8. Thompson A.P., Glandt E.D., Macromolecules, 1996, 29, 4314.

9. Madden W.G., J. Chem. Phys., 1995, 102, 5572.

10. Madden W.G., J. Chem. Phys., 1995, 103, 8156. 
11. Chandler D., J. Phys.: Condens. Matter, 1991, 3, F1.

12. Given J.A., Stell G., J. Chem. Phys., 1992, 97, 4573.

13. Given J.A. Stell G., Physica A, 1994, 209, 495.

14. Given J.A., J. Chem. Phys., 1995, 102, 2934.

15. Lomba E., Given J.A., Stell G., Weis J.J., Levesque D., Phys. Rev. E, 1993, 48, 233.

16. Meroni A., Levesque D., Weis J.J., J. Chem. Phys., 1996, 105, 1101.

17. Alvarez M., Levesque D., Weis J.J., Phys. Rev. E, 1999, 60, 5495.

18. Fernaud M.J., Lomba E., Weis J.J., Phys. Rev. E, 2001, 64, 051501.

19. Schöll-Paschinger E., Levesque D., Weis J.J., Kahl G., Phys. Rev. E, 2001, 64, 011502.

20. Fernaud M.J., Lomba E., Martin C., Levesque D., Weis J.J., J. Chem. Phys., 2003, 119, 364.

21. Fernaud M.J., Lomba E., Lee L.L., J. Chem. Phys., 1999, 111, 10275.

22. Kaminsky R.D., Monson P.A., J. Chem. Phys., 1991, 95, 2936.

23. Vega C., Kaminsky R.D., Monson P.A., J. Chem. Phys., 1993, 99, 3003.

24. Rosinberg M.L., Tarjus G., Stell G., J. Chem. Phys., 1994, 100, 5172.

25. Kierlik E., Rosinberg M.L., Tarjus G., Monson P., J. Chem. Phys., 1995, 103, 4256.

26. Krakoviack V., Kierlik E., Rosinberg M.L., Tarjus G., Monson P., J. Chem. Phys., 2001, 115, 11289.

27. Dong W., Kierlik E., Rosinberg M.L., Phys. Rev. E, 1994, 50, 4750.

28. Dong W., J. Chem. Phys., 1995, 102, 6570.

29. Brennan J.K., Dong W., J. Chem. Phys., 2002, 116, 8948.

30. Dong W., Chen X.S., Zheng W.M., Phys. Rev. E, 2005, 72, 012201.

31. Zhao S.L., Dong W., Liu Q.H., J. Chem. Phys., 2006, 125, 244703.

32. Dong W., Krakoviack V., Zhao S.L., J. Phys. Chem. B (submitted).

33. Zhao S.L., Dong W., Liu Q.H., J. Chem. Phys. (in press).

34. Van Tassel P.R., Talbot J., Viot P., Tarjus G., Phys. Rev. E, 1997, 56, R1299.

35. Van Tassel P.R., J. Chem. Phys., 1997, 107, 9530.

36. Van Tassel P.R., Phys. Rev. E, 1999, 60, R25.

37. Zhang L., Van Tassel P.R., J. Chem. Phys., 2000, 112, 3006.

38. Cheng S., Van Tassel P.R., J. Chem. Phys., 2001, 114, 4974.

39. Sarkisov L., Van Tassel P.R., J. Chem. Phys., 2005, 123, 164706.

40. Bratko D., Chakraborty A.K., Phys. Rev. E, 1995, 51, 5805.

41. Bratko D., Chakraborty A.K., J. Chem. Phys., 1996, 104, 7700.

42. Cardenas M., Tosi M.P., Physica B, 1996, 222, 143.

43. Cardenas M., Tosi M.P., J. Phys.: Condens. Matter, 1997, 9, 2767.

44. Bryk P., Pizio O., Sokolowski S., J. Chem. Phys., 1998, 109, 2310.

45. Domingues H., Hribar Lee B., Vlachy V., Pizio O., Physica A, 2003, 324, 469.

46. Duda Y., Henderson D., Pizio O., Wasan D., J. Phys. Chem. B, 1998, 102, 1562.

47. Duda Y., Patrykiejew A., Pizio O., Sokolowski S., Physica A, 1999, 265, 424.

48. Duda Y., Sokolowski S., Bryk P., Pizio O., J. Phys. Chem. B, 1998, 102, 5490.

49. Duda Y., Pizio O., Sokolowski S., J. Phys. Chem. B, 2004, 108, 19442.

50. Henderson D., Patrykiejew A., Pizio A., Sokolowski S., Physica A, 1996, 233, 67.

51. Hribar B., Pizio O., Trokhymchuk A., Vlachy V., J. Chem. Phys., 1997, 107, 6335.

52. Hribar B., Pizio O., Trokhymchuk A., Vlachy V., J. Chem. Phys., 1998, 109, 2480.

53. Hribar B., Vlachy V., Trokhymchuk A., Pizio O., J. Phys. Chem. B, 1999, 103, 5361.

54. Hribar B., Vlachy V., Pizio O., J. Phys. Chem. B, 2000, 104, 4479.

55. Hribar B., Vlachy V., Pizio O., J. Phys. Chem. B, 2001, 105, 4727.

56. Ilnytsky J., Patrykiejew A., Sokolowski S., Pizio O., J. Phys. Chem. B, 1999, 103, 868.

57. Kovalenko A., Sokolowski S., Henderson D., Pizio O., Phys. Rev. E, 1998, 57, 1824.

58. Kovalenko A., Pizio O., J. Chem. Phys., 1998, 108, 8651.

59. Kovalenko A., Hirata F., J. Chem. Phys., 2001, 115, 8620.

60. Milan Malo B., Pizio O., Trokhymchuk A., Duda Y., J. Colloid and Interface Sci., 1999, 211, 387.

61. Padilla P., Pizio O., Trokhymchuk A., Vega C., J. Phys. Chem. B, 1998, 102, 3012.

62. Padilla P., Vega C., J. Chem. Phys., 1997, 106, 1997.

63. Pizio O., Sokolowski S., Phys. Rev. E, 1997, 56, R63.

64. Pizio O., Renugopalakrishnan V., Trokhymchuk A., J. Colloid and Interface Sci., 1999, 211, 367.

65. Rzysko W., Pizio O., Sokolowski S., Physica A, 1999, 273, 241.

66. Trokhymchuk A., Pizio O., Holovko M., Sokolowski S., J. Phys. Chem., 1996, 100, 17004.

67. Trokhymchuk A., Pizio O., Holovko M., Sokolowski S., J. Chem. Phys., 1997, 106, 200.

68. Vlachy V., Hribar B., Pizio O., Physica A, 2002, 314, 156. 
69. Vlachy V., Domingues H., Pizio O., J. Phys. Chem. B, 2004, 108, 1046.

70. Schmidt M., Phys. Rev. E, 2002, 66, 041108.

71. Schmidt M., Schöll-Paschinger E., Köfinger J., Kahl G., J. Phys.: Condens. Matter, 2002, 14, 12099.

72. Schmidt M., Phys. Rev. E, 2003, 68, 021106.

73. Schmidt M., Brader J.M., J. Chem. Phys., 2003, 119, 3495.

74. Schmidt M., Dijkstra M., J. Chem. Phys., 2004, 121, 12067.

75. Schmidt M., J. Phys.: Condens. Matter, 2005, 2005, 17, S3481.

76. Reich H., Schmidt M., J. Stat. Phys., 2004, 116, 1683.

77. Wessels P.P.F., Schmidt M., Löwen H., Phys. Rev. E, 2003, 68, 061404.

78. Wessels P.P.F., Schmidt M., Löwen H., Phys. Rev. Lett., 2005, 94, 078303.

79. Archer A.J., Schmidt M., Evans R., Phys. Rev. E, 2006, 73, 011506.

80. Lafuente L., Cuesta J.A., Phys. Rev. E, 2006, 74, 041502.

81. Pellecane G., Caccamo C., Wilson D.S., Lee L.L., Phys. Rev. E, 2004, 69, 061202.

82. Sung B.J., Yethiraj A., J. Chem. Phys., 2005, 123, 074909.

83. Widom B., Rowlinson J.S., J. Chem. Phys., 1970, 52, 1670.

\title{
Флюїди в пористих середовищах: ідеальний газ в різних матрицях
}

\author{
В.Донг \\ Лабораторія хімії, Ліонська вища нормальна школа, Франція \\ Отримано 16 серпня 2007 р.
}

На даний час $є$ розвинуті різні моделі для опису адсорбції флюїдів у пористих середовищах. В цій роботі представлено деякі точні аналітичні результати, отримані для ідеального газу в різних пористих середовищах. Показано, що розрахунки не завжди $є$ тривіальними. В деяких випадках складних матриць неможливо отримати жодних аналітичних результатів навіть для ідеального газу.

Ключові слова: пористі матеріали, адсорбовані флюїди

PACS: 61.20.Gy, 61.43.Gt 
